\title{
Boundary tracking algorithms for determining the stability of mode-locked pulses
}

\author{
Shaokang Wang,* Andrew Docherty, Brian S. Marks, and Curtis R. Menyuk \\ Department of Computer Science and Electrical Engineering, University of Maryland, Baltimore County, \\ 1000 Hilltop Circle, Baltimore, Maryland 21250, USA \\ ${ }^{*}$ Corresponding author: swan1@umbc.edu
}

Received August 4, 2014; accepted September 4, 2014;

posted September 24, 2014 (Doc. ID 220362); published October 30, 2014

\begin{abstract}
We develop robust computational methods, referred to as boundary tracking algorithms, that can rapidly determine the existence and stability of pulses in passively mode-locked laser systems over a broad parameter range. Applying the boundary tracking algorithms to the cubic-quintic mode-locking equation, we find a rich dynamical structure. (C) 2014 Optical Society of America

OCIS codes: (000.3860) Mathematical methods in physics; (000.4430) Numerical approximation and analysis; (140.4050) Mode-locked lasers.

http://dx.doi.org/10.1364/JOSAB.31.002914
\end{abstract}

\section{INTRODUCTION}

Passively mode-locked lasers are used to generate ultra-short, high-energy, and stable optical pulses. Compactness, stability, and cost are among the major concerns for practical design of these lasers. The stability of these systems as system parameters vary must be determined to optimize the system and its components.

Despite the vast quantity of both experimental and theoretical work that has been published on passively mode-locked laser systems [1-3], little theoretical work has been done to investigate the stability of these systems over a broad parameter range. Typical theoretical studies solve the evolution equations starting from noise or some other initial conditions and allow the solution to evolve until it either settles down to a stationary or periodically stationary state or fails to settle down after a long evolution time [4-6]. This approach can be ambiguous, since it is often not clear how long it is necessary to wait for a pulse to settle down, and the computation time required to evolve to a steady state approaches infinity in principle when the system parameters approach a stability boundary.

Here we describe a different approach that is based on dynamical systems theory $[7,8]$. A mode-locked pulse is a stationary or periodically stationary solution of a nonlinear dynamical system and can also be viewed as an equilibrium of that system. In this paper we refer to the mode-locked pulse solutions of the equations that we will be studying as equilibrium solutions. If any possible perturbation grows exponentially, then the system is linearly unstable. The stability can be determined by solving a linear eigenvalue problem $[9,10]$. Once a mode-locked solution (an equilibrium) has been found for a single set of parameters using the evolution equations, we can rapidly trace the equilibrium solution as the system parameters vary by solving a root-finding problem to obtain the equilibrium solution without solving the evolution equations. In parallel, we determine the solution's stability as the system parameters vary. Once a stability boundary is encountered, we can then track its location in the parameter space. This approach allows us to rapidly determine the existence and stability of pulses over a broad parameter range.

In this work, we apply this dynamical approach to the oldest and most widely used model equation of a passively modelocked laser system. This model equation was originally proposed by Haus [11], and we refer to it as the Haus mode-locking equation (HME), which may be written as

$$
\begin{aligned}
\frac{\partial u}{\partial z}= & {\left[-i \phi+\frac{g(|u|)}{2}\left(1+\frac{1}{2 \omega_{g}^{2}} \frac{\partial^{2}}{\partial t^{2}}\right)-\frac{l}{2}-\frac{i \beta^{\prime \prime}}{2} \frac{\partial^{2}}{\partial t^{2}}+i \gamma|u|^{2}\right] u } \\
& +f_{\mathrm{sa}}(|u|) u,
\end{aligned}
$$

where $u(t, z)$ is the complex field envelope, $t$ is the retarded time, $z$ is the propagation distance, $\phi$ is the phase rotation per unit length, $l$ is the loss coefficient, $g(|u|)$ is the saturated gain, $\beta^{\prime \prime}$ is the group velocity dispersion coefficient, $\gamma$ is the Kerr coefficient, and $\omega_{g}$ is the gain bandwidth. The value of $\phi$ has no effect on the pulse evolution except to induce on overall phase rotation. It is common to set it to zero, but it physically corresponds to the rate of change of the carrier envelope offset. It is mathematically convenient for us to let it be arbitrary for now. If the relaxation of the laser medium is much slower than the pulse repetition rate, the saturated gain is well approximated by

$$
g(|u|)=g_{0} /\left[1+P_{\mathrm{av}}(|u|) / P_{\mathrm{sat}}\right],
$$

where $g_{0}$ is the unsaturated gain, $P_{\mathrm{av}}(|u|)$ is the average power in the laser cavity, and $P_{\text {sat }}$ is the saturation power of the amplifier. We may write $P_{\text {av }}(|u|)=\int_{-T_{R} / 2}^{T_{R} / 2}|u(t, z)|^{2} \mathrm{~d} t / T_{R}$, where $T_{R}$ is the round-trip time. The function $f_{\mathrm{sa}}(|u|)$ represents the model of fast saturable absorption. In the HME, we have $f_{\text {sa }}(|u|)=\delta|u|^{2}$, where $\delta$ is the fast saturable absorption constant. 
The HME predicts only a narrow stability range for $\delta$ that is inconsistent with what has been observed experimentally [12]. Motivated by this observation-and in an effort to more accurately model the laser physics-other models of the fast saturable absorption have been introduced. A common approach is to add a quintic term, and $f_{\mathrm{sa}}(|u|)$ becomes

$$
f_{\mathrm{sa}}(|u|)=\delta|u|^{2}-\sigma|u|^{4}
$$

where $\sigma>0$ is the coefficient of the quintic term $[4,5,13,14]$, which relaxes the stability constaints posed on the term $\delta$ and enables us to find more stable pulse solutions in a broader range of the parameter space. We refer to the mode-locking equation that is formulated in Eqs. (1)-(3) as the cubic-quintic mode-locking equation (CQME). The CQME is not a quantitatively accurate model of any real passively mode-locked laser system of which we are aware. However, it is simple, and it contains the essential elements that any model of a passively mode-locked laser system must have to ensure the existence of stable pulse solutions. Hence, it has been widely used to obtain qualitative insights into the behavior of many lasers, and is thus a useful model upon which to test our algorithms. At the end of this paper, we briefly discuss how the algorithms must be modified to be applied to more specialized and realistic models.

When dynamical systems go unstable as a parameter varies, the instability mechanisms are referred to in the nonlinear dynamics literature as bifurcations [ㄱ, $\underline{\text { ] }}$. The bifurcations that can occur in our system with the parameters that we are using are saddle-node bifurcations, which occur when the mode-locked pulse amplitude becomes unstable, and Hopf bifurcations, which occur when continuous waves become unstable. In our system, the spectrum of eigenvalues has a discrete component, referred to as the discrete spectrum and a continuous component, referred to as the continuous or essential spectrum $[15,16]$. It is possible in our system for discrete eigenvalues to emerge from the continuous spectrum. These bifurcations are called edge bifurcations [15].

In this paper, we will determine the stability region of the CQME in the parameter space $(\sigma, \delta)$, which are the parameters of the fast saturable absorption, keeping other parameters fixed. We operate in the anomalous dispersion regime, in which $\beta^{\prime \prime}<0$. In Section 2 , we review the properties of the bifurcations that appear in our study (saddle-node, Hopf, and edge). In Section 3, we describe the equilibrium solutions to the CQME, derive the eigenvalue equation that governs the stability of these solutions, and present the eigenvalue spectrum in a typical stable case. In Section $\underline{4}$ we present the computational techniques that we use to find the equilibrium solutions as the system parameters vary, determine their stability, and find the stability boundary locations. In Section 5 we present our results. Finally, Section $\underline{6}$ contains the conclusions.

\section{REVIEW OF RELEVANT DYNAMICAL THEORY}

It has been known since the late 19th century that solving the evolution equations is not an effective approach for determining the stability of a nonlinear dynamical system, particularly as the system parameters vary [17]. Instead, it is better to use geometric or dynamical methods in which one first determines stationary or periodically stationary solutions of the dynamical system, referred to as equilibria, and one then linearizes the equation about these equilibria to find their linear stability. This basic approach then becomes the starting point for addressing more complex issues, such as the nonlinear stability of the system and the onset of chaos; however, these issues are not addressed in this paper.

As noted in Section 1, these powerful dynamical methods have been systematically exploited in many areas of science and engineering_-including fluids and plasmas $[\underline{18}, \underline{19}]$ and, in recent years, biological systems $[7,8]$. However, these methods have not been systematically applied to mode-locked lasers. To our knowledge, they have only been applied in special cases where analytical solutions are known for the equilibria $[\underline{15}, \underline{20}]$.

The basic approach that we will use is to first find an equilibrium solution for one set of parameters using the evolution equations. We can then determine the equilibrium solution as parameters vary by solving a root-finding problem, which is far more computationally efficient than is solving the evolution equations, especially when the point of operation in parameter space approaches the stability boundary. Additionally, solving the root-finding problem allows us to find equilibria regardless of their stability. We vary parameters until we encounter a stability boundary, and we then move along the boundary-tracking or mapping its location. The stability is determined by calculating the eigenvalues of the linearized evolution equations. When any of the eigenvalues has a positive real part, then the equilibrium is unstable. At a stability boundary in the problem that we are considering in this paper, one or more eigenvalues whose real parts are negative become pure imaginary. At that point, one of two things can happen as the parameters vary further. First, the equilibrium can continue to exist, but some of the eigenvalues of the linearized evolution equation become positive. In this case, the equilibrium is unstable. Alternatively, the equilibrium can cease to exist. In either case, this behavior is referred to in the mathematics literature as a bifurcation $[7,8]$.

In the problem that we will be addressing in this paper, we will encounter two types of bifurcations when our system becomes unstable. The first type is a saddle-node bifurcation. This type of bifurcation can be illustrated with the first-order ordinary differential equation (ODE)

$$
\frac{\mathrm{d} x}{\mathrm{~d} t}=-r+x^{2},
$$

where both $x$ and $r$ are real. When $r>0$, this equation has two equilibrium solutions, $x \equiv x_{0}= \pm r^{1 / 2}$. The equilibrium $x_{0}=$ $r^{1 / 2}$ is unstable, while the equilibrium $x_{0}=-r^{1 / 2}$ is stable. When $r=0$, the two equilibria coincide, and, in a sense, "annihilate" each other [7], so that when $r<0$, there is no longer an equilibrium. In this case, the eigenvalue of the linearized equation for the stable equilibrium is real and negative. The system becomes unstable at the point that the eigenvalue becomes equal to zero.

The second type of bifurcation is a Hopf bifurcation. In this case, two eigenvalues that are complex conjugates simultaneously cross the imaginary axis. This type of bifurcation can be illustrated with the second-order equation 


$$
\begin{aligned}
& \dot{x}=\mu x-\omega y+\alpha\left(x^{2}+y^{2}\right) x, \\
& \dot{y}=\omega x+\mu y+\beta\left(x^{2}+y^{2}\right) y,
\end{aligned}
$$

where $\mu, \omega, \alpha$, and $\beta$ are all parameters of the system. This system has an equilibrium at $(x, y)=(0,0)$ for any parameter values. The linearized equation has the eigenvalues $\lambda=\mu \pm i \omega$. When the parameter $\mu$ change from negative to positive-unlike the case of saddle-node bifurcation where the equilibrium disappears when the bifurcation occursthe equilibrium continues to exist, but becomes unstable.

There is a third type of bifurcation that we will encounter in this paper. Equation (4) is a partial differential equation on the infinite line, i.e., the retarded time $t$ extends from $-\infty$ to $\infty$. The corresponding linearized equation will have both a discrete and continuous spectrum. It is possible, as parameters vary, for new discrete eigenvalues to appear. This sort of bifurcation is called an edge bifurcation in the mathematics literature [21]. A relatively simple, linear illustration of this behavior is the well-known three-slab waveguide as the index of refraction of the intermediate slab varies. This waveguide may be described by the equation [22]

$$
\frac{\mathrm{d}^{2} u}{\mathrm{~d} x^{2}}+\left[k_{0}^{2} \Delta n^{2}-\lambda^{2}\right] u=0,
$$

where $k_{0}$ is the wavenumber of the light, $\Delta n^{2}$ is the difference between the squared indices of refraction of the central and the two outside slabs, and $\lambda$ is the eigenvalue. When $\Delta n^{2}<0$, then there is no discrete spectrum. Only a continuous spectrum exists with purely imaginary eigenvalues, $\lambda$. By contrast, when $\Delta n^{2}>0$, there is at least one discrete eigenvalue that is purely real. This eigenvalue bifurcates out of the continuous spectrum, starting at the point where $\Delta n^{2}=0$.

\section{THE EQUILIBRIUM SOLUTION AND ITS STABILITY}

In this paper, we will find the stability boundary in the $(\sigma, \delta)$ plane while keeping other system parameters fixed. The first step in determining the stability boundaries as system parameters $(\sigma, \delta)$ vary is to seek a stationary pulse solution of the CQME $\left[u_{0}(t), \phi_{0}\right]$ in the form of

$$
u(t, z)=u_{0}(t)
$$

so that $u(t, z)$ is independent of $z$. We note that $\phi=\phi_{0}$ is not arbitrary in this equilibrium solution, but must be found in parallel with $u_{0}(t)$. The equilibrium solution $u_{0}(t)$ is an equilibrium (or fixed point) of the dynamical system that exists only for a special value of $\phi_{0}$. Analytical solutions of the CQME exist in certain parameter regimes. However, the known exact analytical solutions are of limited use because they exist only for limited combinations of the coefficients of the CQME [ㅁ, 12$]$, and they are unstable when $\beta^{\prime \prime}<0$. We provide more detail on these analytical solutions in Appendix A. Recently, an interesting class of approximate solutions that are called highly chirped solitons has been reported [23-27]. However, in this paper we do not consider such solutions since our study focuses on the anomalous dispersion regime, while the reported approximate analytical solutions are found in the normal dispersion regime. In general, we are able to find stable numerical solutions in regions where the known analytical solutions do not exist, and it is this more general case in which we are interested.

To determine the linear stability of the system once $\left[u_{0}(t), \phi_{0}\right]$ has been found for a given set of parameters, we linearize Eq. (1) and obtain

$$
\begin{aligned}
\frac{\partial \Delta u}{\partial z}= & {\left[-i \phi_{0}-\frac{l}{2}+\frac{g\left(\left|u_{0}\right|\right)}{2}\left(1+\frac{1}{2 \omega_{g}^{2}} \frac{\partial^{2}}{\partial t^{2}}\right)-\frac{i \beta^{\prime \prime}}{2} \frac{\partial^{2}}{\partial t^{2}}\right.} \\
& \left.+2(i \gamma+\delta)\left|u_{0}\right|^{2}-3 \sigma\left|u_{0}\right|^{4}\right] \Delta u \\
& -\frac{g^{2}\left(\left|u_{0}\right|\right)}{g_{0} T_{R} P_{\mathrm{sat}}} \operatorname{Re}\left[\left\langle u_{0}, \Delta u\right\rangle\right]\left(1+\frac{1}{2 \omega_{g}^{2}} \frac{\partial^{2}}{\partial t^{2}}\right) u_{0} \\
& +\left[(i \gamma+\delta) u_{0}^{2}-2 \sigma\left|u_{0}\right|^{2} u_{0}^{2}\right] \Delta u^{*}
\end{aligned}
$$

where $\Delta u(t, z)=u(t, z)-u_{0}(t)$ is a small perturbation of $u_{0}(t)$, and $\left\langle u_{0}, \Delta u\right\rangle=\int_{-T_{R} / 2}^{T_{R} / 2} u_{0}^{*} \Delta u \mathrm{~d} t$. By taking $\Delta u(t, z)=$ $\exp (\lambda z) \Delta u(t)$, where $\lambda$ is a constant, we obtain an eigenvalue problem:

$$
\lambda \Delta u=\frac{\partial}{\partial z} \Delta u,
$$

where $\lambda$ is an eigenvalue and $\Delta u$ is the eigenmode corresponding to $\lambda$. The linear stability of the equilibrium solution can be determined by the distribution of all eigenvalues on the complex plane, as discussed in Section 2. A dynamical system that is linearly unstable can in principle be nonlinearly stable because the system evolves rapidly to a nearby equilibrium or limit cycle $[7,8]$. However, it is usually found in practice that linear stability is a prerequisite for stable behavior. In this paper, we are concerned only with linear stability, and we will refer to linearly stable and unstable systems simply as "stable" and "unstable."

In order to illustrate the behavior when a stable equilibrium exists for which no analytical solution is known, we consider the parameter set that is shown in Table 1 . Except for $\sigma$ and $\delta$, all parameters will be held fixed with these values throughout this paper. In Fig. 1 , we set $(\sigma, \delta)=(0.002,0.035)$, and we show the evolution of an initial pulse $u(t=0, z)=$ $0.2 \exp \left[-(t / 5)^{2}\right]$. In Fig. 2 , we show the final equilibrium solution $u_{0}(t)$, where the phase at $t=0$ is set equal to zero, and we find $\phi_{0}=1.856$. There is a non-zero chirp, which we indicate using false color. We will describe in more detail the computational procedure that we use to find this solution in the next section.

In Fig. 3, we show the linearized eigenvalue spectrum. The spectrum includes two branches that are symmetric about the real axis and four real discrete eigenvalues that correspond physically to perturbations of the equilibrium solutions central time $\left(\lambda_{t}\right)$, central phase $\left(\lambda_{\phi}\right)$, amplitude $\left(\lambda_{a}\right)$, and central frequency $\left(\lambda_{f}\right)[\underline{28}, \underline{29}]$. The real parts of all the eigenvalues are

Table 1. Normalized Values of Parameters

\begin{tabular}{lccc}
\hline Parameter & Value & Parameter & Value \\
\hline$g_{0}$ & 0.4 & $T_{R} P_{\text {sat }}$ & 1 \\
$l$ & 0.2 & $\omega_{g}$ & $\sqrt{10} / 2$ \\
$\gamma$ & 4 & $\beta^{\prime \prime}$ & -2 \\
\hline
\end{tabular}




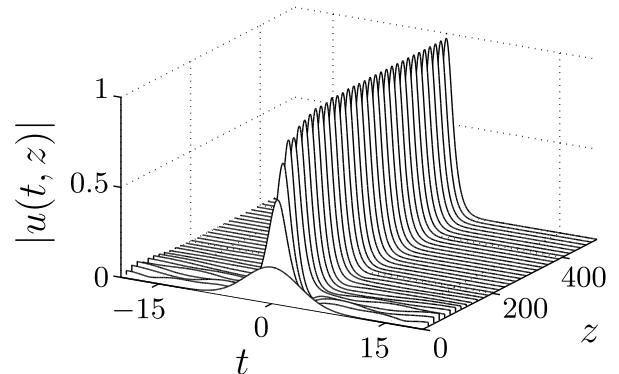

Fig. 1. When $\sigma=0.002$ and $\delta=0.035$, starting with an initial pulse $u(t=0, z)=0.2 \exp \left[-(t / 5)^{2}\right]$, the system evolves to a final equilibrium solution $u_{0}(t)$

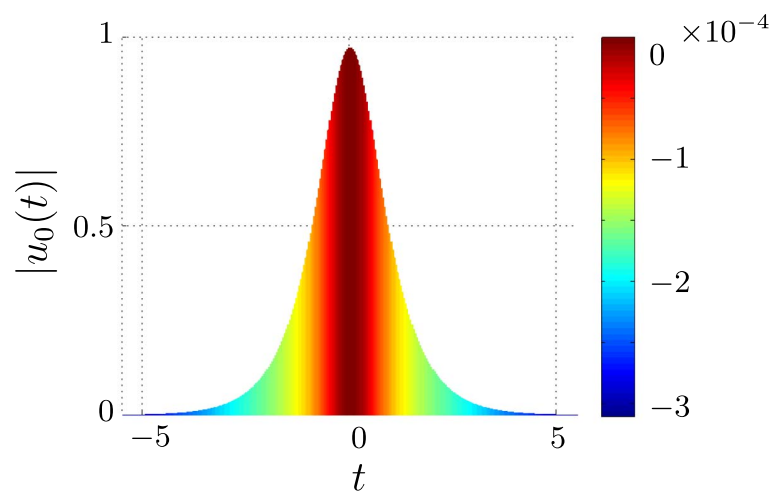

Fig. 2. Equilibrium solution $u_{0}(t)$ for the case $(\sigma, \delta)=(0.002,0.035)$. The false color indicates the phase of the pulse in radians.

negative except for $\lambda_{t}$ and $\lambda_{\phi}$, which equal 0 . These eigenvalues must equal zero because the CQME, as well as its linearization, Eq. (8), are invariant with respect to time and phase shifts. One consequence is that these eigenvalues must remain strictly zero as the system parameters vary and so cannot lead to instability. Another consequence is that the equilibria are only neutrally stable. In particular, noise perturbations will lead to random, unconstrained fluctuations in the central time and phase of the mode-locked pulses. One of the great advances in mode-locked laser technology in the past 15 years has been the development of electronic feedback systems that can lock the central time and central phase of the modelocked pulse to an external reference [30]. From a mathematical perspective, these feedback systems break the invariance of the CQME, leading to coupled systems of equations, describing the coupled optical-electronic systems, which when linearized about their equilibrium solutions have eigenvalues

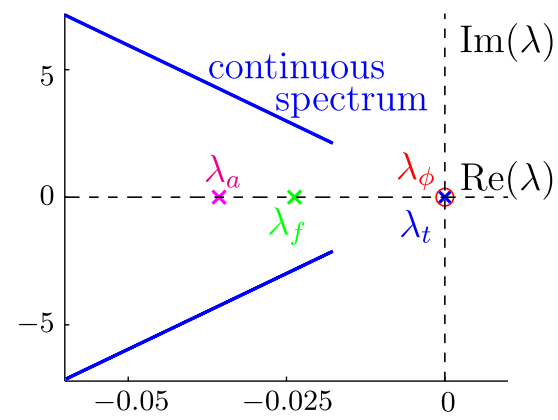

Fig. 3. Spectrum of the eigenvalue problem of Eqs. (8) and (9) at the stable equilibrium solution of the CQME when $(\sigma, \delta)=(0.00 \overline{2}, 0.035)$. whose real parts are all strictly negative [31]. A detailed discussion of this behavior is outside the scope of this paper.

The eigenvalue spectrum in Fig. 3 is qualitatively the same as the spectrum that appears in soliton perturbation theory, in which the equilibria are soliton solutions of the nonlinear Schrödinger equation [28,29]. In that case, there are also two complex conjugate branches of the continuous spectrum and four discrete eigenvalues. We have found that this behavior is generic in the $(\sigma, \delta)$ plane, with the other parameters given in Table 1 , until $\delta$ becomes relatively large, at which point edge bifurcations appear. The parameters in Table 1 correspond to the anomalous dispersion regime; so, it is not surprising that the eigenvalue spectrum should correspond closely to the soliton spectrum. In preliminary studies, we have found that the spectrum changes significantly when the average dispersion becomes normal.

\section{DESCRIPTION OF THE ALGORITHMS}

We now describe in detail the boundary tracking algorithms that we use to determine the stability boundaries in the $(\sigma, \delta)$ plane. This algorithm is really a collection of algorithms that carry out the following tasks:

1. Solution of the evolution equations. We must solve the evolution equations to find a stable equilibrium (mode-locked) solution for at least one set of parameters, as described in Section 2. This stable equilibrium is then the starting point for the remainder of the algorithm. We also solve the evolution equations on occasion to check our results and determine the evolution of unstable solutions. To solve the evolution equations, we use a variant of the split-step method that we have described elsewhere [32]. We have verified that this approach is both robust and computationally efficient.

2. Track the equilibrium solution as parameters vary. At equilibrium, we have $\partial u / \partial z=0$ in Eq. (1), and we find that Eq. (1) becomes

$$
\begin{aligned}
& {\left[-i \phi_{0}-\frac{l}{2}+\frac{g\left(\left|u_{0}\right|\right)}{2}\left(1+\frac{1}{2 \omega_{g}^{2}} \frac{\mathrm{d}^{2}}{\mathrm{~d} t^{2}}\right)-\frac{i \beta^{\prime \prime}}{2} \frac{\mathrm{d}^{2}}{\mathrm{~d} t^{2}}\right.} \\
& \left.+(i \gamma+\delta)\left|u_{0}\right|^{2}-\sigma\left|u_{0}\right|^{4}\right] u_{0}=0,
\end{aligned}
$$

where $\left[u_{0}(t), \phi_{0}\right]$ denotes the equilibrium solution, and $g\left(\left|u_{0}\right|\right)$ is given by Eq. (2). The equilibrium solution is subject to the boundary condition $u_{0}(t) \rightarrow 0$ as $t \rightarrow \pm \infty$. In our computation, this boundary condition is essentially equivalent to a Dirichlet boundary condition or a periodic boundary condition because we use a time window such that $u_{0}(t) \approx 0$ at the edge of the time window. The setup of such a time window will be described in detail in Section 4.A. The determination of $\left[u_{0}(t), \phi_{0}\right]$ is essentially a nonlinear root-finding problem. Starting from an already-determined solution $\left[u_{0}\left(t ; \sigma_{1}, \delta_{1}\right)\right.$, $\left.\phi_{0}\left(\sigma_{1}, \delta_{1}\right)\right]$, we can determine a nearby solution $\left[u_{0}\left(t ; \sigma_{2}, \delta_{2}\right)\right.$, $\left.\phi_{0}\left(\sigma_{2}, \delta_{2}\right)\right]$ using a variant of Newton's method, and continue to find solutions along a path in the $(\sigma, \delta)$ plane. We discuss this algorithm in Section 4.A.

3. Tracking the stability boundaries of the continuous waves. We determine the stability of each equilibrium solution by solving the linearized eigenvalue equations, Eqs. (8) and (9). The approach is different for the discrete spectrum and the continuous spectrum. The continuous spectrum 
corresponds to modes with an infinite extent. The eigenmodes outside the limited time window in which the equilibrium pulse exists will be complex exponentials, and the pulse will affect the eigenvalue only through its effect on $g(|u|)$. We take advantage of that to find the continuous spectrum. When the continuous spectrum touches the imaginary axis, a Hopf bifurcation occurs, and the equilibrium becomes unstable. We vary both $\sigma$ and $\delta$ along paths that are parallel to the stability boundary, where these paths exist in both the stable and the unstable regions, and then we interpolate to find the boundary. We describe these algorithms in Section 4.B.1.

4. Tracking the stability boundaries of the discrete eigenmodes. The discrete spectrum corresponds to eigenmodes that have a limited extent in the time domain, so we can accurately determine the eigenmodes and the eigenvalues using a limited time window. We typically find a stability boundary by varying $\delta$ with a fixed value of $\sigma$ until we encounter a value at which $\lambda_{a}$ becomes zero or the root-finding procedure fails to converge to a pulse solution. When $\lambda_{a}$ becomes zero, a saddlenode bifurcation occurs, and the equilibrium disappears. We then vary both $\delta$ and $\sigma$ along three paths that run parallel to the stability boundary and use three points to extrapolate to the boundary values. We describe these algorithms in Section 4.B.2.

5. Find the edge bifurcations and track the corresponding stability boundary. We have found that when $\delta$ becomes sufficiently large $(\delta \approx 9.5)$, then an edge bifurcation occurs, and two new complex conjugate discrete eigenvalues appear. These eigenvalues then cross over the imaginary axis, and the corresponding equilibrium becomes unstable via a Hopf bifurcation. As $\delta$ continue to increase, a whole series of edge bifurcations take place. The exact location of the first edge bifurcation and the Hopf bifurcations in $(\sigma, \delta)$ are difficult to calculate since the eigenmodes have a temporal extent that is much broader than the equilibrium pulses when the Hopf bifurcation takes place. If the linearized equation was an ODE, we could solve the problem in a time window of limited extent using shooting methods, but that is not possible because the linearized equation is an integro-differential equation that is non-local in time. We instead formulate the problem as an overdetermined boundary value problem with exponentially decaying solutions as $t \rightarrow \pm \infty$ and we search for the values of $(\sigma, \delta)$ at which a solution exists. We describe these algorithms in Section 4.B.3.

\section{A. Solving for the Equilibrium Solution}

For a given set of parameters $(\sigma, \delta)$, we find the equilibrium solution $\left[u_{0}\left(t ; \sigma_{1}, \delta_{1}\right), \phi_{0}\left(\sigma_{1}, \delta_{1}\right)\right]$ by solving the nonlinear root-finding problem given by Eq. (10) and the gain saturation equation, Eq. (2). However, the dependence of the saturated gain $g$ on the unknown function $u_{0}(t)$ leads to a Jacobian that is a dense matrix, which we must avoid for computational efficiency. Hence, we rewrite Eq. (2) as

$$
g-g_{0} /\left[1+P_{\mathrm{av}}\left(\left|u_{0}\right|\right) / P_{\text {sat }}\right]=0,
$$

where $g$ is treated as an unknown variable.

We define a time window with a duration $T$ in which the pulse solution differs significantly from zero. We form a vector of time points after discretization, $\mathbf{t}=\left[t_{1}, t_{2}, \ldots, t_{j}, \ldots, t_{N}\right]^{T}$, where $t_{1}=-T / 2, \quad t_{j}=t_{1}+(j-1) \delta t, \quad j=1,2, \ldots, N, \quad$ and $\delta t=T / N$. The function $u(z, t)$ is represented by a column vector $\mathbf{u}$ of length $N$, where $\mathbf{u}_{j}(z)$ is the computational approximation of $u\left(z, t=t_{j}\right)$. As is usual in computational studies, we vary $T$ and $N$ as the parameters vary to be certain that both are sufficiently large to have no discernible effect on the computational results. An extended discussion of this issue is provided in Appendix $\underline{\mathrm{B}}$. We typically set $N=512$ and set $T$ somewhere between $40 \tau$ and $60 \tau$, where $\tau$ is the duration of the equilibrium pulse, which is obtained using [33]

$$
\tau^{2}=\frac{\int_{-T / 2}^{T / 2}\left(t-t_{0}\right)^{2}\left|u_{0}(t)\right|^{2} \mathrm{~d} t}{\int_{-T / 2}^{T / 2}\left|u_{0}(t)\right|^{2} \mathrm{~d} t}
$$

where $t_{0}$ is the geometric center of the pulse:

$$
t_{0}=\frac{\int_{-T / 2}^{T / 2} t\left|u_{0}(t)\right|^{2} \mathrm{~d} t}{\int_{-T / 2}^{T / 2}\left|u_{0}(t)\right|^{2} \mathrm{~d} t}
$$

To obtain an explicit Jacobian for the system that is composed of Eqs. (10) and (11), another computational difficulty is that $|u|$ is not differentiable since the complex conjugates explicitly appear. To resolve this issue, we form an extended system by splitting the system into its real and imaginary parts. We let $u_{0}(t)=v(t)+i w(t)$ and $u_{0, j}=v_{j}+i w_{j}$, where $v(t)$ and $w(t)$ are the real and the imaginary components of $u_{0}(t)$, and $v_{j}(z), w_{j}(z)$, and $u_{0, j}$ are the corresponding discretizations. When discretized, we can combine Eqs. (ㅇ) and (11) and formulate the following root-finding problem:

$$
\begin{aligned}
& (g-l) \mathbf{v}+g /\left(2 \omega_{g}^{2}\right) \mathbf{v}_{t t}+2 \phi \mathbf{w}+\beta^{\prime \prime} \mathbf{w}_{t t}+\mathbf{p}=\mathbf{0}, \\
& \quad-2 \phi \mathbf{v}-\beta^{\prime \prime} \mathbf{v}_{t t}+(g-l) \mathbf{w}+g /\left(2 \omega_{g}^{2}\right) \mathbf{w}_{t t}+\mathbf{q}=\mathbf{0}, \\
& g\left(P_{0}+\|\mathbf{v}+i \mathbf{w}\|^{2}\right)-g_{0} P_{0}=0,
\end{aligned}
$$

where $\mathbf{v}_{t t}$, and $\mathbf{w}_{t t}$ are vectors that represent the second-order differentiation in $t$ of $v$ and $w$, respectively, and $P_{0}=$ $T_{R} P_{\text {sat }} /(\delta t)$. Provided that $T$ and $N$ are set to be large enough, the vectors $\mathbf{v}_{t t}$ and $\mathbf{w}_{t t}$ can be evaluated using the fast Fourier transform (FFT) since $u_{0}(t)$ is a smooth function. We define the zero vector $\mathbf{0}$, whose components are all $0 \mathrm{~s}$, and we define the vector norm $\|\mathbf{x}\|$, so that $\|\mathbf{x}\|=\sqrt{\mathbf{x}^{H} \mathbf{x}}$, where $\mathbf{x}^{H}$ is the complex conjugate transpose of $\mathbf{x}$. We define the vector $\mathbf{p}$ and $\mathbf{q}$, whose $j$ th components are given by

$$
\begin{aligned}
& p_{j}(\mathbf{v}, \mathbf{w})=2\left(\delta v_{j}-\gamma w_{j}\right)\left(v_{j}^{2}+w_{j}^{2}\right)-2 \sigma v_{j}\left(v_{j}^{2}+w_{j}^{2}\right)^{2} \\
& q_{j}(\mathbf{v}, \mathbf{w})=2\left(\gamma v_{j}+\delta w_{j}\right)\left(v_{j}^{2}+w_{j}^{2}\right)-2 \sigma w_{j}\left(v_{j}^{2}+w_{j}^{2}\right)^{2} .
\end{aligned}
$$

The unknowns of the ODE system of Eq. (14) are a composite vector $[\mathbf{v}, \mathbf{w}, g, \phi]^{T}$, and the Jacobian is

$$
\left[\begin{array}{cccc}
{\left[g /\left(2 \omega_{g}^{2}\right)\right] \mathrm{D}_{t}^{2}+\mathrm{P}_{v}} & \beta^{\prime \prime} \mathrm{D}_{t}^{2}+\mathrm{P}_{w} & \mathbf{v}+\mathbf{v}_{t t} / \omega_{g}^{2} & \mathbf{w} \\
\mathbf{Q}_{v}-\beta^{\prime \prime} \mathrm{D}_{t}^{2} & {\left[g /\left(2 \omega_{g}^{2}\right)\right] \mathrm{D}_{t}^{2}+\mathrm{Q}_{w}} & \mathbf{w}+\mathbf{w}_{t t} / \omega_{g}^{2} & -\mathbf{v} \\
2 g \mathbf{v}^{T} & 2 g \mathbf{w}^{T} & P_{0}+\|\mathbf{u}\|^{2} & 0
\end{array}\right],
$$

where $D_{t}^{2}$ is the second-order finite difference matrix. Taking both accuracy and computation efficiency into consideration, we use a seven-point central finite-difference scheme as well as periodic boundary conditions, so that 


$$
\mathrm{D}_{t}^{2}=\frac{1}{(\delta t)^{2}}\left[\begin{array}{llllllllll}
c_{0} & c_{1} & c_{2} & c_{3} & 0 & \cdots & 0 & c_{3} & c_{2} & c_{1} \\
c_{1} & c_{0} & c_{1} & c_{2} & c_{3} & 0 & \cdots & 0 & c_{3} & c_{2} \\
c_{2} & c_{1} & c_{0} & c_{1} & c_{2} & c_{3} & 0 & \cdots & 0 & c_{3} \\
c_{3} & c_{2} & c_{1} & c_{0} & c_{1} & c_{2} & c_{3} & 0 & \cdots & 0 \\
0 & c_{3} & c_{2} & c_{1} & c_{0} & c_{1} & c_{2} & c_{3} & 0 & \vdots \\
\vdots & \ddots & \ddots & \ddots & \ddots & \ddots & \ddots & \ddots & \ddots & 0 \\
0 & \cdots & 0 & c_{3} & c_{2} & c_{1} & c_{0} & c_{1} & c_{2} & c_{3} \\
c_{3} & 0 & \cdots & 0 & c_{3} & c_{2} & c_{1} & c_{0} & c_{1} & c_{2} \\
c_{2} & c_{3} & 0 & \cdots & 0 & c_{3} & c_{2} & c_{1} & c_{0} & c_{1} \\
c_{1} & c_{2} & c_{3} & 0 & \cdots & 0 & c_{3} & c_{2} & c_{1} & c_{0}
\end{array}\right],
$$

where $c_{0}=-49 / 18, c_{1}=1.5, c_{2}=-0.15$, and $c_{3}=1 / 90$. The matrices $\mathrm{P}_{v}, \mathrm{P}_{w}, \mathrm{Q}_{v}$, and $\mathrm{Q}_{w}$ are diagonal matrices whose components are dependent on $\phi, \mathbf{v}$, and $\mathbf{w}$ in the following way:

$$
\begin{aligned}
P_{v, j}(\phi, \mathbf{v}, \mathbf{w})= & (g-l)-4 \gamma v_{j} w_{j}+6 \delta v_{j}^{2}+2 \delta w_{j}^{2} \\
& -10 \sigma v_{j}^{4}-2 \sigma w_{j}^{4}-12 \sigma v_{j}^{2} w_{j}^{2}, \\
P_{w, j j}(\phi, \mathbf{v}, \mathbf{w})= & 2 \phi-2 \gamma v_{j}^{2}-6 \gamma w_{j}^{2}+4 \delta v_{j} w_{j} \\
& -8 \sigma v_{j}^{3} w_{j}-8 \sigma v_{j} w_{j}^{3}, \\
Q_{v, j j}(\phi, \mathbf{v}, \mathbf{w})= & -2 \phi+2 \gamma w_{j}^{2}+6 \gamma v_{j}^{2}+4 \delta v_{j} w_{j} \\
& -8 \sigma v_{j} w_{j}^{3}-8 \sigma v_{j}^{3} w_{j}, \\
Q_{w, j j}(\phi, \mathbf{v}, \mathbf{w})= & (g-l)+4 \gamma v_{j} w_{j}+6 \delta w_{j}^{2}+2 \delta v_{j}^{2} \\
& -10 \sigma w_{j}^{4}-2 \sigma v_{j}^{4}-12 \sigma v_{j}^{2} w_{j}^{2} .
\end{aligned}
$$

This Jacobian is a non-square matrix with a dimension of $(2 N+2) \times(2 N+1)$; therefore, we cannot solve the system of Eq. (14) using the standard Newton's method and must instead solve it in a least-square sense. We have found that the Levenberg-Marquart method works well [34].

To track the equilibrium efficiently over a broad range of parameters, the initial guess for $\left[u_{0}\left(t ; \sigma_{2}, \delta_{2}\right), \phi_{0}\left(\sigma_{2}, \delta_{2}\right)\right]$ of the root-finding problem is set to be an equilibrium solution $\left[u_{0}\left(t ; \sigma_{1}, \delta_{1}\right), \phi_{0}\left(\sigma_{1}, \delta_{1}\right)\right]$ that has been found previously at the point $\left(\sigma_{1}, \delta_{1}\right)$, where $\left(\sigma_{2}, \delta_{2}\right)$ is close to the point $\left(\sigma_{1}, \delta_{1}\right)$ in the parameter space, as noted in the description of task 2 in Section 4.

\section{B. Boundary Tracking Algorithms}

The stability of the pulse solution is determined by the ODE in Eq. (8). In our computations, we continue to split the system into two components, as the complex conjugate operations explicitly appear. We define $\Delta u, \Delta v$, and $\Delta w$ as the perturbations of the equilibrium solution $u_{0}$ and its real and imaginary parts, $v_{0}$ and $w_{0}$, respectively. Using the same discretization as in Section 4.A, we linearize Eq. (9) to obtain the linear eigenvalue problem

$$
\lambda\left[\begin{array}{c}
\Delta \mathbf{v} \\
\Delta \mathbf{w}
\end{array}\right]=\frac{\mathrm{d}}{\mathrm{d} z}\left[\begin{array}{c}
\Delta \mathbf{v} \\
\Delta \mathbf{w}
\end{array}\right] \approx \mathrm{J}\left[\begin{array}{c}
\Delta \mathbf{v} \\
\Delta \mathbf{w}
\end{array}\right] .
$$

Note that in Eq. (17), the vectors $\Delta \mathbf{v}$ and $\Delta \mathbf{w}$ are not necessarily real since we do not split $\lambda$ into its real part and imaginary part, so that the eigenmodes $\Delta v$ and $\Delta w$ are possibly complex functions. In Eq. (17), the quantity $\mathrm{J}$ is the Jacobian of the ODE system of Eq. (8),

$$
\mathrm{J}=\frac{1}{2}\left[\begin{array}{ll}
\mathrm{J}_{11} & \mathrm{~J}_{12} \\
\mathrm{~J}_{21} & \mathrm{~J}_{22}
\end{array}\right],
$$

and the blocks of $\mathrm{J}$ are given by

$$
\begin{aligned}
& \mathrm{J}_{11}=\mathrm{P}_{v}+\frac{g}{2 \omega_{g}^{2}} \mathrm{D}_{t}^{2}-\mathrm{G}_{f}^{\prime}\left(\mathbf{v}_{0}, \mathbf{v}_{0}\right), \\
& \mathrm{J}_{12}=\mathrm{P}_{w}+\beta^{\prime \prime} \mathrm{D}_{t}^{2}-\mathrm{G}_{f}^{\prime}\left(\mathbf{v}_{0}, \mathbf{w}_{0}\right), \\
& \mathrm{J}_{21}=\mathrm{Q}_{v}-\beta^{\prime \prime} \mathrm{D}_{t}^{2}-\mathrm{G}_{f}^{\prime}\left(\mathbf{w}_{0}, \mathbf{v}_{0}\right), \\
& \mathrm{J}_{22}=\mathrm{Q}_{w}+\frac{g}{2 \omega_{g}^{2}} \mathrm{D}_{t}^{2}-\mathrm{G}_{f}^{\prime}\left(\mathbf{w}_{0}, \mathbf{w}_{0}\right),
\end{aligned}
$$

where $\mathrm{G}_{f}^{\prime}$ is the derivative of the function $g(\mathbf{x}, \mathbf{y})$ with respect to $\mathbf{x}$ or $\mathbf{y}$ :

$$
\mathrm{G}_{f}^{\prime}(\mathbf{x}, \mathbf{y})=\frac{2 g^{2}}{g_{0} N P_{0}}\left(\mathrm{I}+\frac{\mathrm{D}_{t}^{2}}{2 \omega_{g}^{2}}\right)\left(\mathbf{x y}^{T}\right) .
$$

In Eqs. (19) and (20), the variables $\mathrm{P}_{v}, \mathrm{P}_{w}, \mathrm{Q}_{v}, \mathrm{Q}_{w}$, and $\mathrm{G}_{f}^{\prime}$ are all evaluated at the equilibrium: $\phi=\phi_{0}, \mathbf{v}=\mathbf{v}_{0}$, and $\mathbf{w}=\mathbf{w}_{0}$. Similarly, we evaluate $g$ as

$$
g=\frac{g_{0}}{1+\left(\mathbf{v}_{0}^{T} \mathbf{v}_{0}+\mathbf{w}_{0}^{T} \mathbf{w}_{0}\right) /\left(N P_{\text {sat }}\right)} .
$$

The eigenvalue spectrum for the case $\sigma=0.002, \delta=0.035$ is shown in Fig. 3. For this case, to accurately track the location of the discrete eigenvalues, the operator $D_{t}^{2}$ should be formulated spectrally [35]. There are cases where edge bifurcations occur and discrete modes emerge from the continuous spectra. We will need a different approach to accurately find those eigenvalues. More details will be discussed in the following subsections.

\section{Continuous Spectrum}

The modes of the continuous spectrum represent perturbations that do not vanish as $t \rightarrow \infty$. There are two branches of the continuous spectrum, as seen in Fig. 3, and they add ripples to the equilibrium solution [36]. The tips of these two branches of eigenvalues will hit the imaginary axis when there is not enough amplitude-dependent loss to suppress the continuous waves, as illustrated in Fig. 4. The continuous waves then grow. This type of instability is called an essential singularity $[15,16]$. An essential instability of the CQME occurs, for example, when $(\sigma, \delta)=(0.002,0.005)$. We can

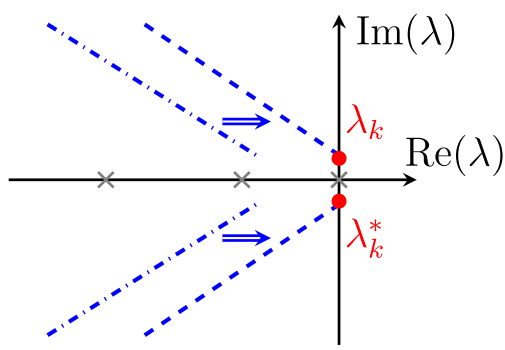

Fig. 4. Illustration of the variation of eigenvalues distribution where the continuous spectrum is unstable. 


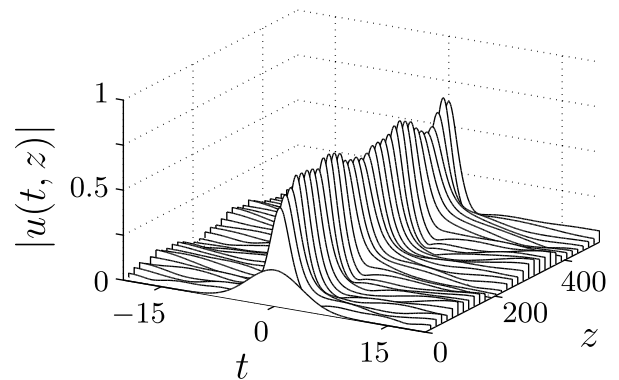

Fig. 5. When $\sigma=0.002$ and $\delta=0.005$, starting with an initial pulse $u(t=0, z)=0.2 \exp \left[-(t / 5)^{2}\right]$, the system never reaches a steady state (equilibrium).

observe this instability in Fig. $\underline{5}$, where an initial pulse does not evolve to any steady state, and its envelope continually fluctuates and exhibits a chaotic-appearing behavior. The unstable equilibrium solution, which we show in Fig. 6 , was obtained using the procedure that is described in Section 4.A. The spectrum of Eq. (17) at this equilibrium is shown in Fig. 7 , where one can see that the continuous spectrum extends into the right half of the complex plane.

Since the modes of the continuous spectrum extend to $t= \pm \infty$, their eigenvalues are most easily determined by studying the modes in that limit, so that $u_{0}(t) \rightarrow 0$. The Jacobian of Eq. (8), if evaluated in the frequency domain, becomes

$$
\mathrm{J}=\frac{1}{2}\left[\begin{array}{cc}
g-l-g \omega^{2} /\left(2 \omega_{g}^{2}\right) & \phi-\beta^{\prime \prime} \omega^{2} \\
-\phi+\beta^{\prime \prime} \omega^{2} & g-l-g \omega^{2} /\left(2 \omega_{g}^{2}\right)
\end{array}\right]
$$

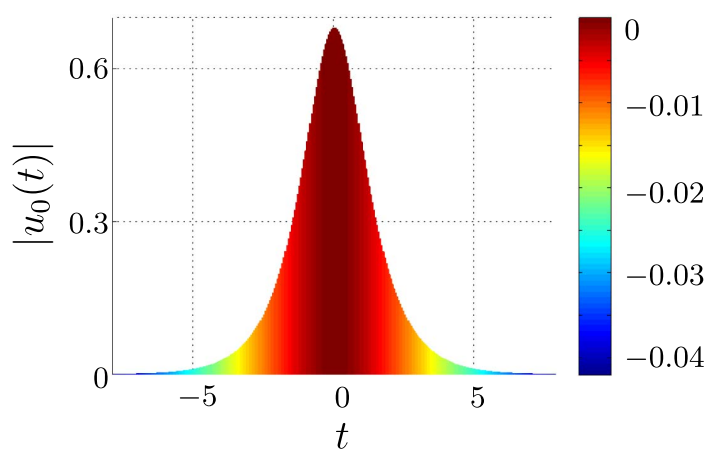

Fig. 6. Equilibrium solution $u_{0}(t)$ for the case $(\sigma, \delta)=(0.002,0.005)$. False color indicates the phase of the pulse in radians.

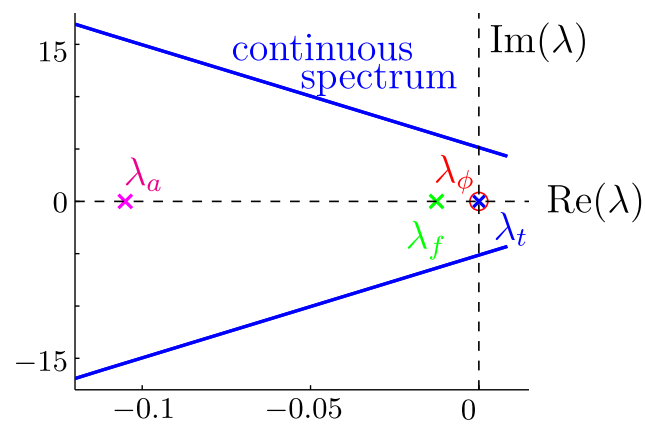

Fig. 7. Eigenvalue spectrum corresponding to the equilibrium solution of the CQME when $(\sigma, \delta)=(0.002,0.005)$. and the eigenvalues of Eq. (ㅁ) are given by

$$
\lambda_{ \pm}=\frac{1}{2}\left[g-l-g \frac{\omega^{2}}{2 \omega_{g}^{2}} \pm i\left(\phi-\beta \omega^{2}\right)\right],
$$

where $\omega$ is the frequency. The stability criterion is that the eigenvalues with the largest real parts, $\lambda_{k}$ and $\lambda_{k}^{*}$, corresponding to $\omega=0$ in Eq. (23), should have a negative real part. We then find the stability condition is

$$
\operatorname{Re} \lambda_{k}=\frac{g-l}{2}<0 \text {. }
$$

We can determine the stability of radiation modes without finding the eigenvalues of the Jacobian computationally, because the saturated gain $g$ can be explicitly calculated once the equilibrium solution is found. The spectrum of the system of Eq. (8) is shown in Fig. 7, where the continuous spectrum is found using Eq. (23).

While the computational solution of Eq. (17) will also yield an estimate of the eigenvalues of the continuous spectrum, we have found that the finite time window leads to inaccurate estimates even when the window is as large as 100 times the pulse duration. As a consequence, it is not computationally feasible to determine the eigenvalues of the continuous spectrum using this numerical approach. The approach that we are using effectively assumes that we have a single pulse in an infinite time window, which is appropriate if the round-trip time $T_{R}$ is sufficiently large compared to the pulse duration $\tau$. In passively mode-locked fiber lasers with a single pulse in the cavity, this ratio is $10^{5}$ or larger and this assumption is reasonable. However, this assumption becomes invalid for mode-locked lasers with high repetition rates or microresonators. A more detailed discussion of this issue may be found in Appendix B.

We implement the boundary tracking algorithm in this case by first varying the cubic coefficient $\delta$, while the quintic coefficient $\sigma$ remains fixed $\left(\sigma=\sigma_{k}\right)$, and determining the variation of the $\lambda_{k}$. Eventually, we encounter a case $p_{1}=\left(\sigma_{1}, \delta_{1}\right)$ in which we cross the stability boundary, as shown schematically in Fig. 8(a), indicating that the corresponding equilibrium solution has become unstable via a Hopf bifurcation. We may then use two nearby stable points, here denoted by $p_{2}=\left(\sigma_{k}, \delta_{2}\right)$ and $p_{3}=\left(\sigma_{k}, \delta_{3}\right)$, to find the boundary using quadratic interpolation. At a nearby value of $\sigma\left(\sigma_{k+1}\right)$, we once again find one unstable and two stable points and again interpolate to find the stability boundary. From these two points on the stability boundary, we obtain an estimate for the slope of the boundary $\mathrm{d} \delta / \mathrm{d} \sigma$, which allows us to predict where the
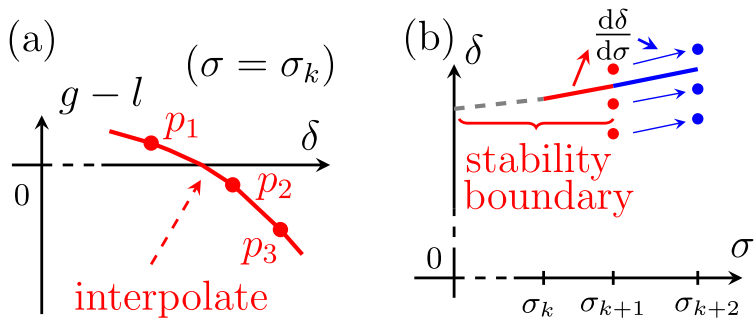

Fig. 8. Boundary tracking algorithm for radiation modes: (a) finding the stability boundary for the case when $\sigma=\sigma_{k}$, and (b) tracking the stability boundary from $\sigma=\sigma_{k}$ to $\sigma=\sigma_{k+1}$. 
three points surrounding the boundary will be when $\sigma=\sigma_{k+2}$. We quadratically interpolate to find the boundary at $\sigma=\sigma_{k+1}$, we correct these predictions, and we obtain a new prediction for the slope. In this way, we accurately and rapidly map out the entire boundary. In this paper, the spacing between the $\delta$ values for extrapolation is about 0.001 , and we change $\sigma$ by 0.002 when tracking this stability boundary. These choices yield a good balance between accuracy and efficiency.

\section{Discrete Spectrum}

As noted previously, the discrete spectrum consists of four eigenvalues until $\delta$ becomes quite large. Two of them, which correspond to time and phase translations $\left(\lambda_{t}\right.$ and $\left.\lambda_{\phi}\right)$, are necessarily equal to zero. One of them, which corresponds to a frequency shift $\left(\lambda_{\omega}\right)$, is always negative; the corresponding eigenmode never goes unstable because of our assumption of a parabolic gain profile. By contrast, the eigenmode that corresponds to a change in the pulse amplitude and energy does go unstable when the nonlinear gain becomes too large to be overcome by the lossy terms in Eq. (1). When that occurs, the corresponding eigenvalue $\left(\lambda_{a}\right)$ crosses through zero, as shown schematically in Fig. 9. This instability has been described in detail for the HME [15], in which case the solution quickly grows, as shown in Fig. 10, and eventually "blows up." This instability has been referred to as the exploding soliton instability in the mode-locked laser literature, and the instability limit is derived in [15] and occurs at $\delta=0.0348$. In the CQME, the quintic loss term that equals $-\sigma|u|^{4} u$ saturates the nonlinear growth, so that the CQME is expected to have an enlarged stability region in the $(\sigma, \delta)$ parameter space. That is indeed the case. We find that as long as $\sigma>0$, there is a stable solution until $\delta \approx 9.5$, almost a factor of 280 greater than the HME's stability limit, indicating that the behavior is qualitatively, not just quantitatively, changed by the addition

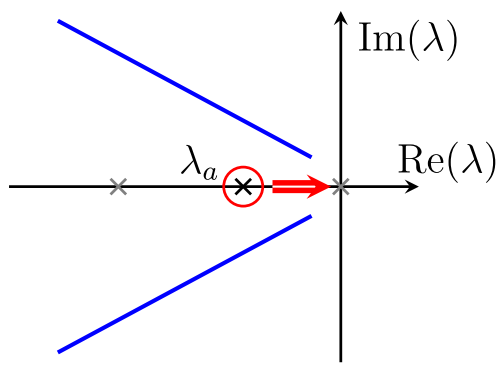

Fig. 9. When a saddle-node bifurcation due to the amplitude eigenmode occurs, the amplitude eigenvalue $\lambda_{a}$ approaches 0 .

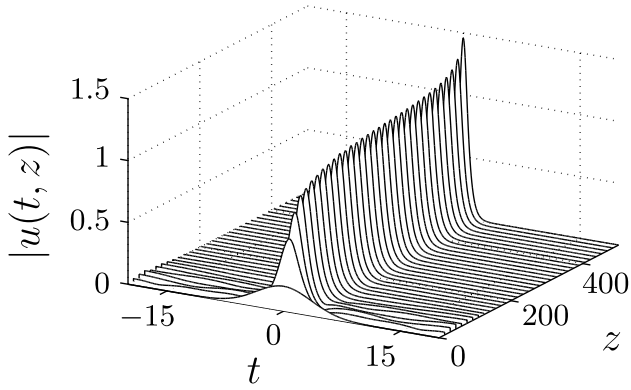

Fig. 10. When $\sigma=0$ and $\delta=0.035$, the peak of the initial pulse $u(t=$ $0, z)=0.2 \exp \left[-(t / 5)^{2}\right]$ grows exponentially as the pulse evolves.
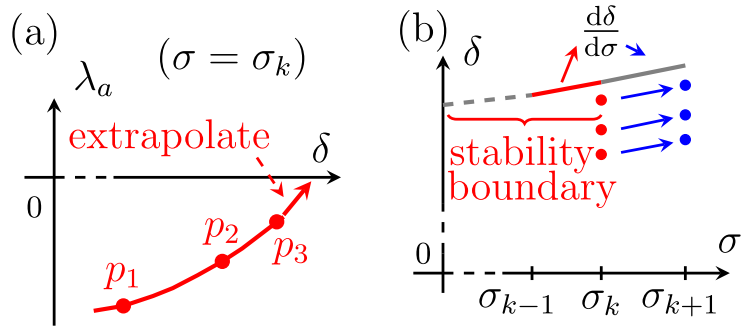

Fig. 11. Boundary tracking algorithm for the amplitude eigenmode: (a) finding the stability boundary for the case $\sigma=\sigma_{k}$ and (b) tracking the stability boundary from $\sigma=\sigma_{k}$ to $\sigma=\sigma_{k+1}$.

of the quintic term. It is possible to demonstrate that an equilibrium solution exists for arbitrary small, positive values of $\sigma$ at values of $\delta$, such as $\delta=6$, that are far larger than the stability limit of the HME. We will publish this result separately.

The amplitude eigenmode becomes unstable via a saddlenode bifurcation so that the equilibrium ceases to exist when, given a fixed value of $\sigma$, the value of $\delta$ changes beyond the value at which $\lambda_{a}=0$. As a consequence, we must modify the boundary tracking algorithm that we used to find the stability boundary for the continuous spectrum. Instead of using quadratic interpolation, we use quadratic extrapolation, following three trajectories in the stable region, as shown schematically in Fig. 11. Otherwise, the algorithm is the same. A typical spacing between the $\delta$ values that we use in our extrapolation is about 0.0001 , and we typically change $\sigma$ by 0.0004 when tracking the boundary. We adjust the spacings of both $\delta$ and $\sigma$ as system parameters vary in order to balance the convergence rate of the root-finding procedure and the computational efficiency.

Another point worth noting is that one should use a spectral differentiation scheme for $D_{t}^{2}$ in the Jacobian of Eq. (19) when computing $\lambda_{a}$ and the other eigenvalues that correspond to the eigenmodes that are well confined in the time window $T$ [9]. Otherwise, the computation of these eigenvalues is either inaccurate or inefficient. With this choice, the operator $D_{t}^{2}$ becomes a dense and symmetric Toeplitz matrix [37]:

$$
\mathrm{D}_{t}^{2}=\frac{4 \pi^{2}}{T^{2}}\left[\begin{array}{cccccc}
d_{0} & d_{1} & d_{2} & \cdots & d_{N-2} & d_{N-1} \\
d_{1} & d_{0} & d_{1} & \ddots & & d_{N-2} \\
d_{2} & d_{1} & \ddots & \ddots & \ddots & \vdots \\
\vdots & \ddots & \ddots & \ddots & d_{1} & d_{2} \\
d_{N-2} & & \ddots & d_{1} & d_{0} & d_{1} \\
d_{N-1} & d_{N-2} & \cdots & d_{2} & d_{1} & d_{0}
\end{array}\right]
$$

where the entries are

$$
\begin{aligned}
d_{0} & =-\left(N^{2}+2\right) / 12, d_{k}=(-1)^{k-1} \csc ^{2}(k \pi / N) / 2, k \\
& =1, \ldots, N-1 .
\end{aligned}
$$

We use the MATLAB routine eig to find the eigenvalues, including $\lambda_{a}$. Since we are interested only in $\lambda_{a}$, we could in principle greatly increase the efficiency of finding $\lambda_{a}$ by using an iterative scheme in which only this eigenvalue is computed. However, the computational time that is required to find $\lambda_{a}$ is in any case small compared to the computational time that is 
required to find the equilibrium solution. So we did not implement this improvement.

\section{Appearance of New Discrete Modes}

As we shall discuss in more detail in Section 5 , the stability boundaries of the continuous modes and the discrete modes are found in the range $0.01<\delta<0.05$ and $0<\sigma<0.01$. When $\delta \approx 9.5$, we find that a pair of new discrete eigenvalues, $\lambda_{e}$ and $\lambda_{e}^{*}$, emerge via an edge bifurcation from the continuous spectrum. Here, we will use $\lambda_{e}$ to denote the new discrete eigenvalue whose imaginary part is positive and $\lambda_{e}^{*}$ to denote its complex conjugate. With a small additional increase in $\delta$, $\Delta \delta \approx 0.001$, the corresponding eigenmodes become unstable via a Hopf bifurcation. We show this process schematically in Fig. 12. As $\delta$ increases, further edge bifurcations occur, so that more discrete eigenmodes appear and then go unstable via Hopf bifurcations.

As $\delta$ continues to increase, so do the real parts of $\lambda_{e}$ and $\lambda_{e}^{*}$ for the first pair of eigenmodes that become unstable, and these corresponding eigenmodes become increasingly narrow. In Figs. 13(a) and 14 we show a comparison of both the equilibrium pulse solutions and the corresponding eigenmodes for $\lambda_{e}$, respectively, when $(\sigma, \delta)=(0.003,9.509)$ and $(\sigma, \delta)=(0.003,13)$. In the first case the eigenmode is stable, while in the second case it is unstable. We note that both $\Delta v$ and $\Delta w$ are even and complex in contrast to the original four discrete eigenmodes for which the eigenmodes corresponding to $\lambda_{t}$ and $\lambda_{\omega}$ are odd and real, while those corresponding to $\lambda_{\phi}$ and $\lambda_{a}$ are even and real. When $\delta=9.509$, which is shortly after the edge bifurcation has occurred, the decay as $|t|$ increases is barely visible. By contrast, the decay is clearly visible when $\delta=13$.

Accurately finding the eigenmodes and eigenvalues that appear right after the edge bifurcation is a difficult computational problem. On one hand, the analytical approach that we used to obtain the continuous spectrum is no longer applicable. On the other hand, as discussed in more detail in Appendix B, very large computational windows are needed to obtain accurate results - too large to be feasible. If the linear eigenvalue problem could be formulated as a differential equation, then we could use shooting methods. However, that is also not possible in this case because of the gain dependence on the pulse energy, so that the linear equation is an integro-differential equation that is non-local in time. We avoid these difficulties by formulating the eigenvalue problem as an overdetermined set of linear equations $\mathcal{L}\left[\lambda_{e}, \Delta u(t) ; \sigma, \delta\right]=0$, where we demand that the solution is exponentially decaying as $t \rightarrow \pm \infty$. Given a pair $(\sigma, \delta)$ and a choice of $\lambda_{e}$ that matches these boundary conditions, the equation $\mathcal{L}=0$ will not, in

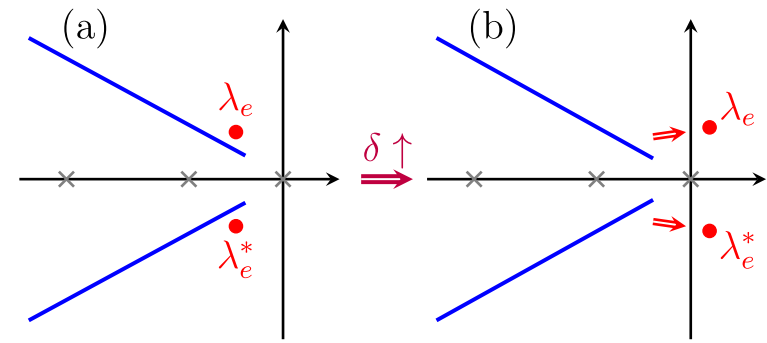

Fig. 12. Illustration of the emergence and destablization of the corresponding eigenmodes of new discrete eigenvalues as $\delta$ grows: from (a) an edge bifurcation to (b) a Hopf bifurcation.

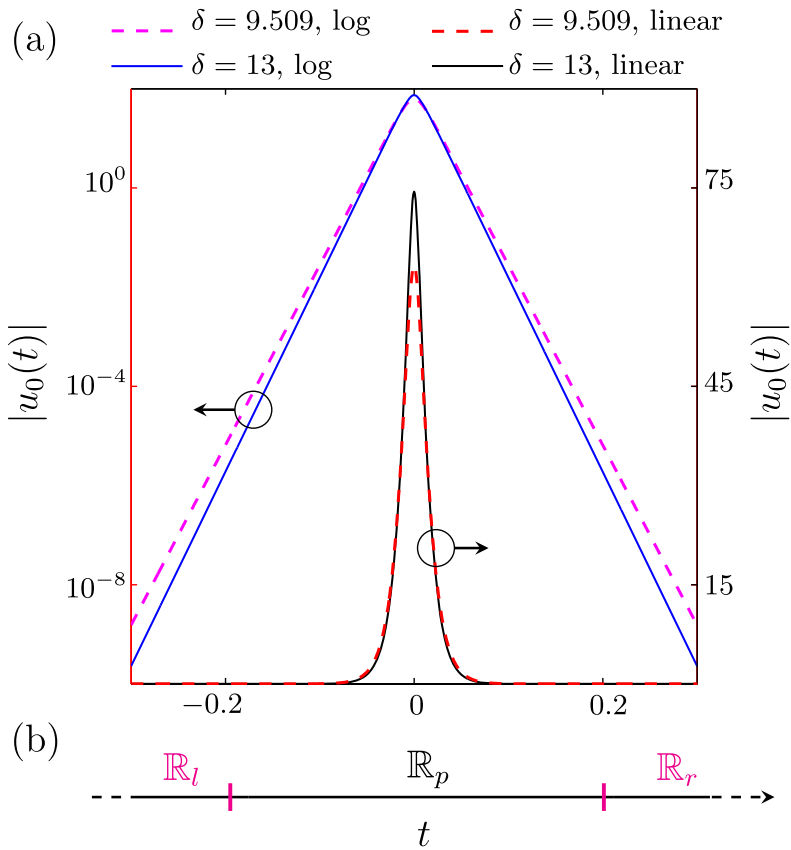

Fig. 13. (a) Amplitudes of the equilibrium pulse solutions when $\delta=9.509$ and $\delta=13$, while the quintic coefficient $\sigma=0.003$ for both. We use $T=0.842$ and $T=0.845$, respectively, in a computation. The pulse amplitudes are shown in both logarithmic and linear coordinates. The amplitudes decay exponentially on the wings as $|t|$ increases. (b) According to the pulse amplitude, we split the computational window into three regions: $\mathbb{R}_{p}$, where the pulse amplitude is significant, as well as $\mathbb{R}_{l}$ and $\mathbb{R}_{r}$, where $u_{0}(t) \approx 0$.

general, have a solution. However, if we have a good initial guess for $\left[\lambda_{e}, \Delta u(t)\right]$, we can find this pair iteratively using a root-finding procedure. We use the secant method, so that we do not need to provide the Jacobian of $\mathcal{L}=0$.

Explicitly, we first combine Eqs. (8) and (9) to obtain

$$
\begin{aligned}
\left(\frac{i \beta^{\prime \prime}}{2}-\frac{g}{4 \omega_{g}^{2}}\right) \frac{\partial^{2} \Delta u}{\partial t^{2}}= & {\left[-\lambda-i \phi_{0}+\frac{g-l}{2}+2(i \gamma+\delta)\left|u_{0}\right|^{2}-3 \sigma\left|u_{0}\right|^{4}\right] \Delta u } \\
& -\frac{g^{2}}{g_{0} T_{R} P_{\mathrm{sat}}} \operatorname{Re}\left[\left\langle u_{0}, \Delta u\right\rangle\right]\left(1+\frac{1}{2 \omega_{g}^{2}} \frac{\mathrm{d}^{2}}{\mathrm{~d} t^{2}}\right) u_{0} \\
& +\left[(i \gamma+\delta) u_{0}^{2}-2 \sigma\left|u_{0}\right|^{2} u_{0}^{2}\right] \Delta u^{*}
\end{aligned}
$$

as well as the conjugate equation for $\Delta u^{*}$, where $g=g\left(\left|u_{0}\right|\right)$. Since $u_{0}(t) \rightarrow 0$ as $t \rightarrow \pm \infty$, we find that Eq. (27) becomes, in this limit,

$$
\left(\frac{i \beta^{\prime \prime}}{2}-\frac{g}{4 \omega_{g}^{2}}\right) \frac{\partial^{2} \Delta u}{\partial t^{2}}=\left(-\lambda-i \phi_{0}+\frac{g-l}{2}\right) \Delta u,
$$

which has a solution $\Delta u(z, t)=C \exp (\eta t+i \lambda z)$, where

$$
\eta^{2}=\frac{g-l-2\left(\lambda+i \phi_{0}\right)}{i \beta^{\prime \prime}-g /\left(2 \omega_{g}^{2}\right)} .
$$

This equation is coupled to a similar equation for $\Delta u^{*}$. When a discrete eigenmode exists and $\lambda=\lambda_{e}$, we can find a solution for which $\operatorname{Re}(\eta)>0$ as $t \rightarrow-\infty$ and $\operatorname{Re}(\eta)<0$ as $t \rightarrow+\infty$. To find this solution computationally, we first obtain $\Delta v$ and $\Delta w$ using the same splitting of $\Delta u$ that we used in Eq. (17). The decay rate of $\Delta v$ and $\Delta w$ as $t \rightarrow \pm \infty$ should equal that of $\Delta u$. 

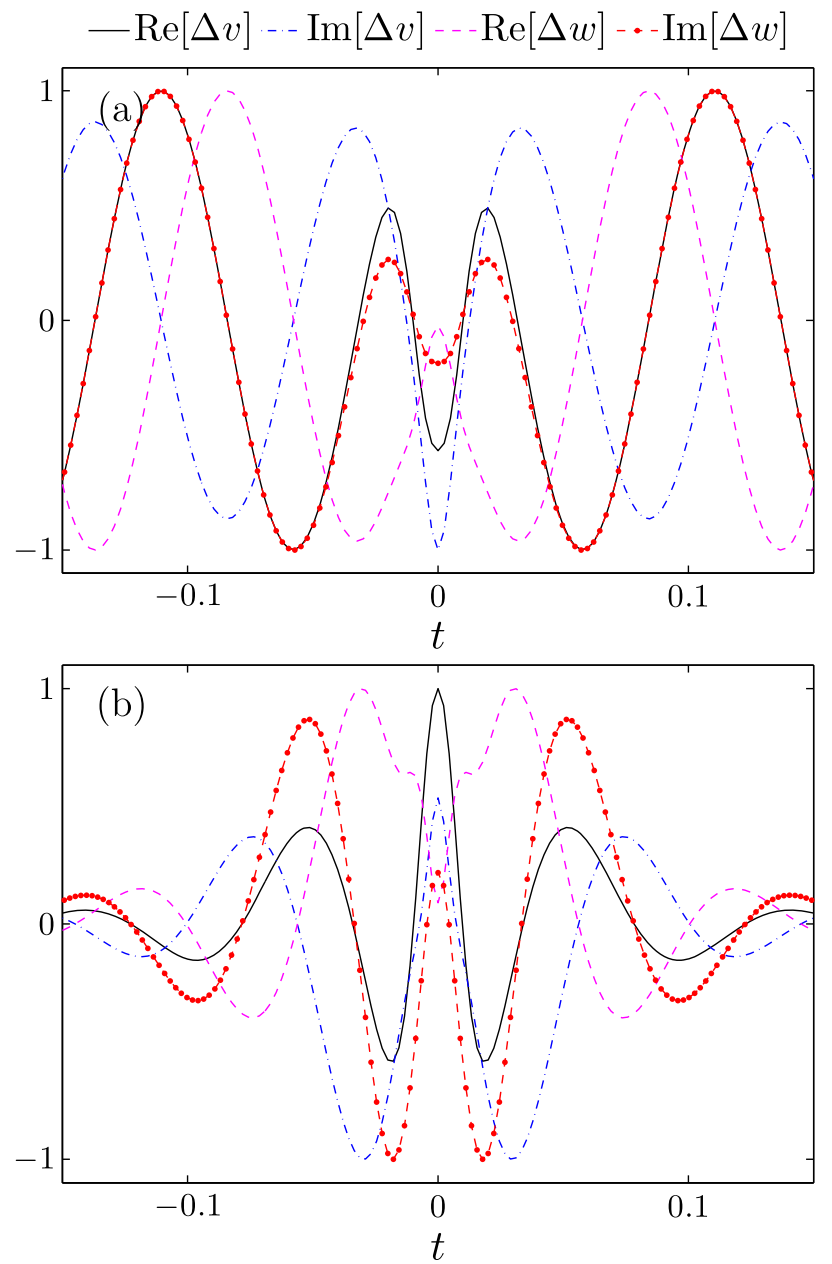

Fig. 14. When $\sigma=0.003$, the shapes of the eigenmodes corresponding to $\lambda_{e}$ : (a) when $\delta=9.509$ and $\lambda_{e}=-0.40+1.05 \times 10^{4} i$, and (b) when $\delta=13$ and $\lambda_{e}=(0.31+1.33) \times 10^{4} i$.

We now split our computational window into three regions, as illustrated in Figs. 13(b) and 15. In the regions denoted $\mathbb{R}_{l}$ and $\mathbb{R}_{r}$ at the left and right edges of the time window, we assume that $u_{0}(t)$ is negligible. We have found that $\left|u_{0}(t)\right|<10^{-6}$ is sufficient in practice. In the region $\mathbb{R}_{l}$ we have

$$
\Delta v=c_{v 1} \exp \left(\eta_{1} t\right)+c_{v 2} \exp \left(\eta_{2} t\right)
$$

$$
\Delta w=c_{w 1} \exp \left(\eta_{1} t\right)+c_{w 2} \exp \left(\eta_{2} t\right)
$$

where

$$
\eta_{1}(\lambda)=\left[-\frac{g-l-2\left(\lambda+i \phi_{0}\right)}{g /\left(2 \omega_{g}^{2}\right)-i \beta^{\prime \prime}}\right]^{1 / 2},
$$

as well as

$$
\eta_{2}(\lambda)=\left[-\frac{g-l-2\left(\lambda-i \phi_{0}\right)}{g /\left(2 \omega_{g}^{2}\right)+i \beta^{\prime \prime}}\right]^{1 / 2},
$$

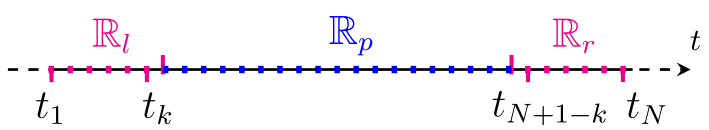

Fig. 15. Illustration of setting $\mathbf{t}$. and we choose the square roots, so that $\operatorname{Re}\left(\eta_{1,2}\right)>0$ and the solution decays as $t \rightarrow-\infty$. We similarly choose solutions for $\Delta v$ and $\Delta w$ in $\mathbb{R}_{r}$ that decay as $t \rightarrow+\infty$. We will only keep elements in the discretized formulation in the region that is labeled $\mathbb{R}_{p}$ in Fig. 15. At the boundaries that are labeled $t_{k}$ and $t_{N+1-k}$ in Fig. 15 , we use the boundary conditions that the solutions are exponentially decaying in accordance with Eq. (30).

When discretized, Eq. (27) becomes

$$
\left[\begin{array}{c}
\mathrm{D}_{t}^{2} \Delta \mathbf{v} \\
\mathrm{D}_{t}^{2} \Delta \mathbf{w}
\end{array}\right]=\mathrm{C}^{-1}(\mathrm{~A}+\mathrm{S})\left[\begin{array}{c}
\Delta \mathbf{v} \\
\Delta \mathbf{w}
\end{array}\right]
$$

where $D_{t}^{2}$ is a second-order difference operator. We use a seven-point difference. The matrices $A$ and $C$ are block-wise diagonal and may be written

$$
\begin{aligned}
& \mathrm{C}=\left[\begin{array}{cc}
g /\left(2 \omega_{g}^{2}\right) \mid & \beta^{\prime \prime} \mid \\
\beta^{\prime \prime} \mid & -g /\left(2 \omega_{g}^{2}\right) \mid
\end{array}\right], \\
& \mathrm{A}=\left[\begin{array}{cc}
-\mathrm{P}_{v}+2 \lambda \mid & -\mathrm{P}_{w} \\
\mathrm{Q}_{v} & \mathrm{Q}_{w}-2 \lambda \mid
\end{array}\right],
\end{aligned}
$$

in which $g, \mathrm{P}_{v}, \mathrm{P}_{w}, \mathrm{Q}_{v}$, and $\mathrm{Q}_{w}$ are evaluated at the equilibrium pulse solution as in Eq. (19), and $\mathrm{S}$ is a dense matrix:

$$
\mathrm{S}=\left[\begin{array}{cc}
\mathrm{G}_{f}^{\prime}\left(\mathbf{v}_{0}, \mathbf{v}_{0}\right) & \mathrm{G}_{f}^{\prime}\left(\mathbf{v}_{0}, \mathbf{w}_{0}\right) \\
-\mathrm{G}_{f}^{\prime}\left(\mathbf{w}_{0}, \mathbf{v}_{0}\right) & -\mathrm{G}_{f}^{\prime}\left(\mathbf{w}_{0}, \mathbf{w}_{0}\right)
\end{array}\right],
$$

where $\mathrm{G}_{f}^{\prime}$ is defined following Eq. (20). Note that in Eqs. (33)(35) and all following equations in this section, the size of the square matrices $\mathrm{D}_{t}^{2}, \mathrm{I}, \mathrm{P}_{v}, \mathrm{P}_{w}, \mathrm{Q}_{v}$, and $\mathrm{Q}_{w}$ is $K \times K$, and the length of the vectors $\mathbf{v}_{0}, \mathbf{w}_{0}, \Delta \mathbf{v}$, and $\Delta \mathbf{v}$ is $K$, where $K=(N-2 k)-\operatorname{not} N$ anymore-as illustrated in Fig. 15, since we consider only the elements of the matrices/vectors that are numbered between $k$ and $N+1-k$. We obtain, from Eq. (33),

$$
\mathrm{M}\left[\begin{array}{c}
\Delta \mathbf{v} \\
\Delta \mathbf{w}
\end{array}\right]=\mathbf{0}
$$

where the matrix $M$ is

$$
\mathrm{M}=\mathrm{C}^{-1}(\mathrm{~A}+\mathrm{S})-\left[\begin{array}{cc}
\mathrm{D}_{t}^{2} & 0 \\
0 & \mathrm{D}_{t}^{2}
\end{array}\right],
$$

which is dependent on $\lambda$ through $A$, and also through $\mathrm{D}_{t}^{2}$, as we will explain later.

Equation (36) may be written as an eigenvalue problem:

$$
\mathrm{M}\left[\begin{array}{c}
\Delta \mathbf{v} \\
\Delta \mathbf{w}
\end{array}\right]=m\left[\begin{array}{c}
\Delta \mathbf{v} \\
\Delta \mathbf{w}
\end{array}\right],
$$

where $m$ is an eigenvalue of M. For Eq. (38), the matrix M is dependent on the value of $\lambda$, so that $m$ is a function of $\lambda, m(\lambda)$. According to Eq. (36), the eigenvalue of interest is the one that yields $m(\lambda)=0$, which is true only if $\lambda=\lambda_{e}$. Therefore, we can treat this eigenvalue problem as a root-finding problem:

$$
(\text { an eigenvalue of } \mathrm{M})=m(\lambda)=0,
$$

where the root is $\lambda=\lambda_{e}$. 
Thus far, we have formulated a root-finding problem based on our previous eigenvalue problem of Eq. (9), and we intend to search for an eigenvalue $\lambda$ that makes $m(\lambda)=0$. We note that the dependence of $m$ on $\lambda$ through $A$ is linear, so that it appears as though Eq. (39) could be solved using the MATLAB routine eig or another linear eigenvalue solution routine. However, we must take the boundary conditions into consideration. The eigenmodes must decay as $t \rightarrow \pm \infty$, which affects our formulation of the matrix $D_{t}^{2}$ as we will describe shortly. Our formulation of $\mathrm{D}_{t}^{2}$ leads to a nonlinear dependence of $m$ on $\lambda$. Therefore, we use the MATLAB routine fsolve to solve this nonlinear root-finding problem iteratively. In each iteration, we find the eigenvalues of $M$ that are close to 0 using the routine eigs.

Before closing this section, we describe our reformulation of $\mathrm{D}_{t}^{2}$ at the boundary of the time window $\mathbb{R}_{p}$ : we cannot use Eq. (15) to build $D_{t}^{2}$ because the periodic boundary conditions that we have been using when finding $u_{0}(t)$ do not apply since $\Delta u(t) \neq 0$ at $t \approx \pm T / 2$, as shown in Fig. 14. Here, we construct $\mathrm{D}_{t}^{2}$ using the boundary conditions of Eq. (30).

Using the seven-point central difference as in Section 4.A, we approximate the second-order differentiation of, for example, $\Delta v$ to $t$, as

$$
\begin{aligned}
\left(\mathrm{D}_{t}^{2} \Delta \mathbf{v}\right)_{l}= & \frac{1}{(\delta t)^{2}}\left[c_{0} \Delta v_{l}+c_{1}\left(\Delta v_{l-1}+\Delta v_{l+1}\right)\right. \\
& \left.+c_{2}\left(\Delta v_{l-2}+\Delta v_{l+2}\right)+c_{3}\left(\Delta v_{l-3}+\Delta v_{l+3}\right)\right]
\end{aligned}
$$

where $1 \leq l \leq K$. We note that the values of $\Delta \mathbf{v}_{n}$, where $n \in\{-2,-1,0, K+1, K+2, K+3\}$, are unknown, but are needed to evaluate $\left(\mathrm{D}_{t}^{2} \Delta \mathbf{v}\right)_{l}$ for $l \in\{1,2,3, K-2, K-1, K\}$. We recall that the eigenmodes can be characterized by Eq. (30) in the region where $u_{0}(t) \approx 0$, which enables us to formulate $\Delta v_{n}$ in terms of $\Delta v_{l}$ and to construct $\mathrm{D}_{t}^{2}$.

We now give an example. We use sub-indices to enumerate the iterations when solving for $m(\lambda)$. Suppose we are at iteration $s$, where $s>1$. We can derive $\eta_{1, s}$ and $\eta_{2, s}$ from $\lambda_{s}$ using Eqs. (31) and (32). Assume we intend to evaluate $\left[\left(\mathrm{D}_{t}^{2}\right)_{s} \Delta \mathbf{v}\right]_{1}$ only from $\Delta v_{1}, \bar{\Delta} v_{2}$, and $\Delta v_{3}$. Using Eq. (30a), we have

$$
\begin{aligned}
& \Delta v_{1}=c_{v 1} \exp \left(\eta_{1, s} t_{1}\right)+c_{v 2} \exp \left(\eta_{2, s} t_{1}\right), \\
& \Delta v_{2}=c_{v 1} \exp \left(\eta_{1, s} t_{2}\right)+c_{v 2} \exp \left(\eta_{2, s} t_{2}\right) .
\end{aligned}
$$

We can determine $c_{v 1}$ and $c_{v 2}$ in terms of $\Delta v_{1, s-1}$ and $\Delta v_{2, s-1}$ as

$$
\left[\begin{array}{l}
c_{v 1} \\
c_{v 2}
\end{array}\right]=\left[\begin{array}{ll}
e^{\eta_{1, s} t_{1}} & e^{\eta_{2, s} t_{1}} \\
e^{\eta_{1, s} t_{2}} & e^{\eta_{2, s} t_{2}}
\end{array}\right]^{-1}\left[\begin{array}{l}
\Delta v_{1, s} \\
\Delta v_{2, s}
\end{array}\right]
$$

Now we write $v_{n}, n=-2,-1,0$ as a combination of $\Delta v_{1, s-1}$ and $\Delta v_{2, s-1}$ using Eq. (30a). For example, $\Delta v_{0}$ can be written as

$$
\Delta v_{0}=\left[\begin{array}{ll}
\exp \left(\eta_{1, s} t_{0}\right) & \exp \left(\eta_{2, s} t_{0}\right)
\end{array}\right]\left[\begin{array}{l}
c_{v 1} \\
c_{v 2}
\end{array}\right]
$$

which, combining with Eq. (42), leads to

$$
\Delta v_{0, s}=p_{1} \Delta v_{1, s-1}+p_{2} \Delta v_{2, s-1},
$$

where $p_{1}=\left[\exp \left(\eta_{1, s} t_{0}+\eta_{2, s} t_{2}\right)-\exp \left(\eta_{2, s} t_{0}+\eta_{1, s} t_{2}\right)\right] / y$, and $p_{2}=\left[-\exp \left(\eta_{1, s} t_{0}+\eta_{2, s} t_{1}\right)+\exp \left(\eta_{2, s} t_{0}+\eta_{1, s} t_{1}\right)\right] / y$, in which $y=\exp \left(\eta_{1, s} t_{1}+\eta_{2, s} t_{2}\right)-\exp \left(\eta_{2, s} t_{1}+\eta_{1, s} t_{2}\right) . \quad$ Likewise, $\quad$ we can write $\Delta v_{-1}$ and $\Delta v_{-2}$ as

$$
\begin{aligned}
& \Delta v_{-1, s}=p_{3} \Delta v_{1, s-1}+p_{4} \Delta v_{2, s-1}, \\
& \Delta v_{-2, s}=p_{5} \Delta v_{1, s-1}+p_{6} \Delta v_{2, s-1},
\end{aligned}
$$

where $p_{3}, p_{4}, p_{5}$, and $p_{6}$ can be determined in a similar fashion as in Eqs. ( $\underline{43})$ and (44) $)$. Now we are able to write $\left[\left(D_{t}^{2}\right)_{s} \Delta \mathbf{v}\right]_{1}$ by combining Eqs. (40), (44), and (45) to obtain

$$
\begin{aligned}
{\left[\left(\mathrm{D}_{t}^{2}\right)_{s} \Delta \mathbf{v}\right]_{1}=} & \frac{1}{(\delta t)^{2}}\left[\left(c_{0}+c_{1} p_{1}+c_{2} p_{3}+c_{3} p_{5}\right) \Delta v_{1, s}\right. \\
& +\left(c_{1}+c_{1} p_{2}+c_{2} p_{4}+c_{3} p_{6}\right) \Delta v_{2, s} \\
& \left.+c_{2} \Delta v_{3, s}+c_{3} \Delta v_{4, s}\right] .
\end{aligned}
$$

Then we can put the coefficients $\Delta v_{1, s}, \Delta v_{2, s}, \Delta v_{3, s}$, and $\Delta v_{4, s}$ in row 1 of $\left(\mathrm{D}_{t}^{2}\right)_{s}$. Rows 2 and 3 of $\left(\mathrm{D}_{t}^{2}\right)_{s}$ can be obtained in a similar fashion, and rows $K, K-1$, and $K-2$ can be found from rows 1,2 , and 3 , respectively, due to the even symmetry. Once $\left(\mathrm{D}_{t}^{2}\right)_{s}$ is constructed, we can construct $\mathrm{M}_{s}$ using Eq. (37), and evaluate its eigenvalue that is closest to 0 and the corresponding eigenvector. These results can then be used to construct $\left(\mathrm{D}_{t}^{2}\right)_{s+1}$.

The algorithm for finding the new discrete eigenvalues via a nonlinear root-find problem is summarized in the following pseudo-code:

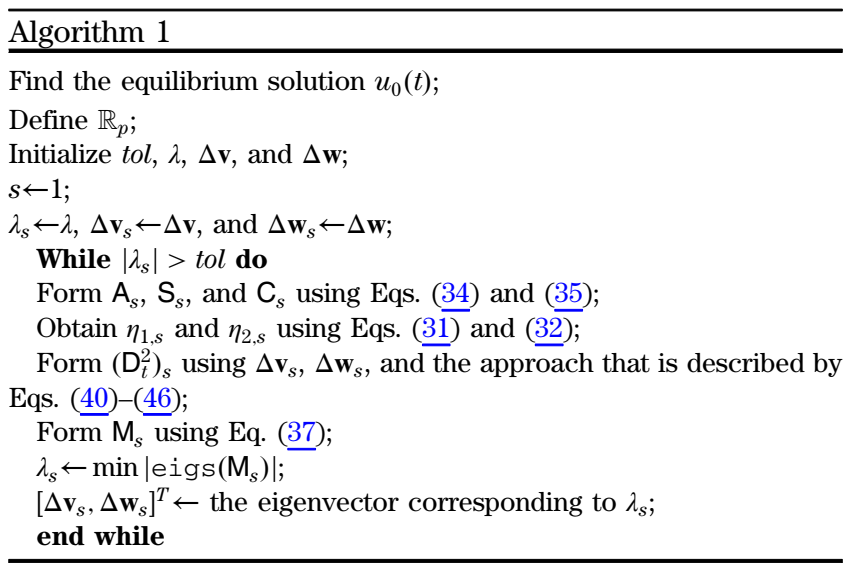

in which tol is the tolerance, i.e., how much $\lambda_{s}$ deviates from 0 . The nonlinear root-finding problem is solved using a fixed-point method in the pseudo-code. In practice, we use the MATLAB routine fsolve to handle the nonlinear root-finding problem in order to obtain faster convergence than is the case when we use the fixed-point method.

The algorithm to track the stability boundary due to edge bifurcations is similar to the one that is illustrated in Fig. 8(a). We obtain $\lambda_{e}(\delta)$ when the iteration converges to $m(\lambda)=\overline{0 \text {; we }}$ track the variation of $\operatorname{Re}\left[\lambda_{e}\right]$ as $\delta$ varies, and we use secondorder polynomial interpolation to find the zero of $\operatorname{Re}\left[\lambda_{e}(\delta)\right]$, since we are still able to find the equilibrium pulse solution even if it is unstable. Then we track the stability boundary as $\sigma$ varies in the same way that is illustrated in Fig. 8(b).

\section{RESULTS}

When $\sigma=0$, the CQME becomes the HME. The equilibrium pulse solution that is given in Eq. (A3) can be obtained 
analytically, and the stability range is $\delta \in[0.01,0.0348]$ [15]. The radiation modes become unstable when $\delta<0.01$ because the nonlinear saturable absorption is too small to stabilize the pulse. The amplitude eigenmode becomes unstable because excessive nonlinear gain causes the pulse solution to blow up, as shown in Fig. 9(b). The boundary tracking algorithm is started from this known case of the HME, and we then gradually increases $\sigma$.

Figure 16 shows the stability regions of the CQME in the parameter space $(\sigma, \delta)$. In general, we have found that the stability regions are characterized by three curves $C_{1}, C_{2}$, and $C_{3}$ in the parameter range that we studied. The blue-hatched region, which is marked $\left\{2_{l}\right\}$, represents the stability region of the solution that is a continuation of the known pulse solution of the HME. We call this solution "the low-amplitude solution." The $\delta$ axis corresponds to the HME solution. Stability region $\left\{2_{l}\right\}$ has the upper bound curve $C_{3}$ and the lower bound curve $C_{1}$. The continuous modes become unstable below $C_{1}$, and this region is labeled $\{1\}$ in Fig. 16. Another pulse solution is stable in the red-hatched region labeled $\left\{2_{h}\right\}$ in Fig. 16 . We call this solution "the high-amplitude solution." Region $\left\{2_{h}\right\}$ has the lower bound curve $C_{2}$, at which the amplitude eigenmode becomes unstable. These lower and the higher amplitude solutions coexist in a triangular-shaped region (labeled $\{3\})$, in which the energy of the higher amplitude solution is always greater than that of the low-amplitude solution, as in the example shown in Fig. 17. The two solutions merge together into a single stable solution in a continuous fashion in region $\left\{2_{h / l}\right\}$, which is colored green. In region $\{1\}$, which is unhatched, the lower-amplitude solution becomes unstable due to the continuum modes.

The instability mechanisms of the lower amplitude solution is similar to that of the HME. Below $C_{1}$ the saturable absorption is too weak to prevent the continuum modes from growing. Meanwhile, the interplay between the cubic and the quintic terms in the fast saturable absorption greatly affects the stability at the boundary of region $\{3\}$. The saddle-node bifurcation appears because the quintic term, $-\sigma|u|^{4} u$, which is lossy, is not able to provide sufficient loss to offset the nonlinear gain that is introduced by the cubic term, $\delta|u|^{2} u$. We see from Fig. 16 that the low-amplitude solution becomes unstable when $\delta$ increases and reaches $C_{3}$, at which the nonlinear gain from the cubic term becomes too large to be compensated by the quintic term. This mechanism resembles the instability mechanism of the HME. However, the high-amplitude solution

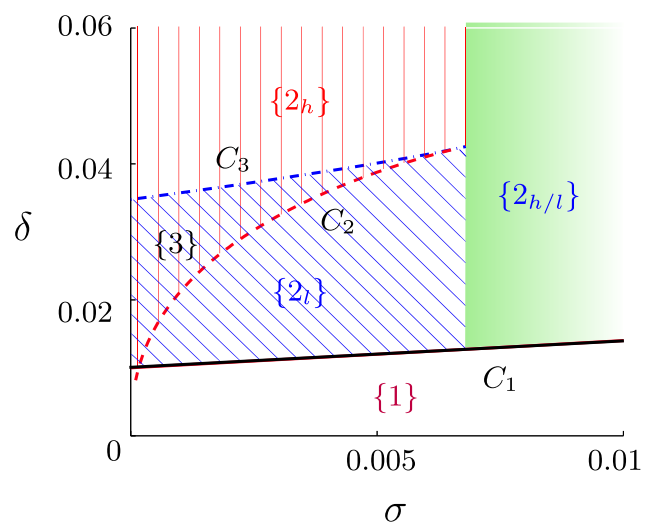

Fig. 16. Stability regions of the CQME. The stability boundaries are marked by three curves, $C_{1}, C_{2}$, and $C_{3}$. becomes unstable when $\delta$ decreases and reaches $C_{2}$. The energy of both solutions grows as $\delta$ increases, and the energy of the high-amplitude solution is greater than that of the lowamplitude solution in region $\{3\}$, so that the former experiences more loss from $-\sigma|u|^{4} u$ than does the latter, which explains why the high-amplitude solution is stable while the low-amplitude solution is unstable on $C_{2}$. As $\delta$ decreases, the energy of the high-amplitude solution becomes smaller, and so is the nonlinear loss induced by the quintic term. Then the high-amplitude solution eventually becomes unstable as $\delta$ keeps decreasing and reaches $C_{2}$ because the nonlinear loss from the quintic term becomes too small to be able to offset the nonlinear gain from the cubic term.

The amplitude instability occurs for the high-amplitude solution on $C_{2}$, while it occurs for the low-amplitude solution on $C_{3}$. Figure 18 shows the variation of the amplitude eigenvalue $\lambda_{a}$ for both solutions near $C_{2}$ and $C_{3}$ for different values of $\sigma$. As $\delta$ increases, for the case $\sigma=0.005$ and $\sigma=0.006$, the eigenvalue $\lambda_{a}$ of the low-amplitude solution grows and eventually approaches 0 . However, the eigenvalue $\lambda_{a}$ of the

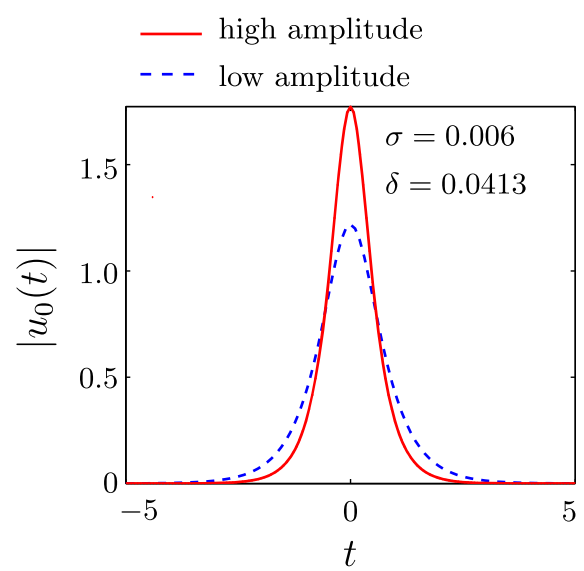

Fig. 17. The two coexisting equilibrium pulse solutions for the case $\sigma=0.006, \delta=0.0413$

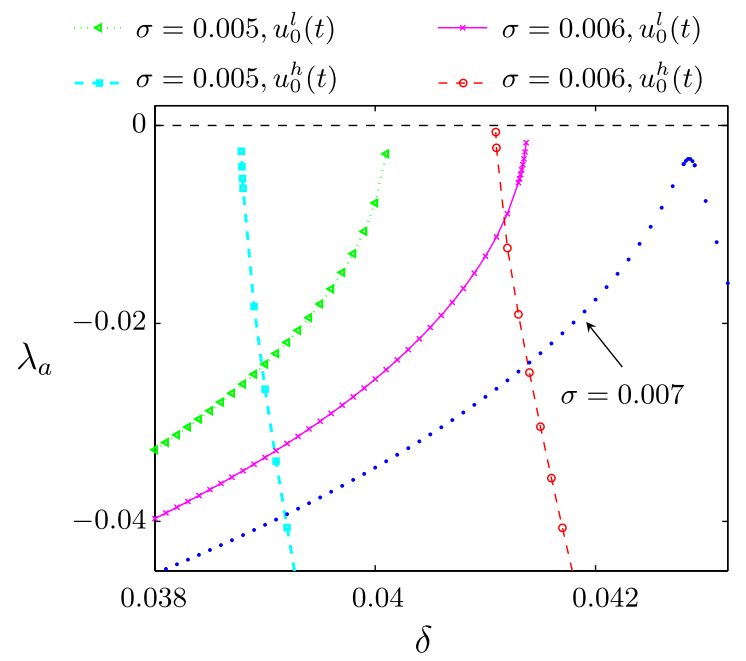

Fig. 18. Variation of the amplitude eigenvalues of the two equilibrium solutions, the low-amplitude solution $u_{0}^{l}(t)$ and the highamplitude solution $u_{0}^{h}(t)$, near curves $C_{2}$ and $C_{3}$ as in Fig. 16. For the case $\sigma=0.007$, we have one single equilibrium solution. 
high-amplitude solution grows and approaches 0 as $\delta$ decreases. For both solutions, we find that $\delta$ at the instability threshold when $\sigma=0.006$ is greater than that when $\sigma=0.005$. However, the separation between the stability boundaries of these two solutions decreases when $\sigma$ increases and becomes zero at $\sigma=0.068$, as shown in Fig. $\underline{16}$, at which point the two solutions merge into a single stable solution. In Fig. 18 we see that when $\sigma=0.007$, the eigenvalue $\lambda_{a}$ first increases as $\delta$, but then starts to decrease before it reaches 0 .

For any position in region $\{3\}$, there are two equilibrium solutions. Which equilibrium solution ultimately appears depends on the specific initial condition. An example is shown in Fig. 19, which corresponds to $(\sigma, \delta)=(0.006,0.0413)$ in region $\{3\}$. The CQME evolves to the low-amplitude solution, which has lower energy than the high-amplitude solution, if we start from an initial pulse $0.8 u_{0}^{h}(t)$, where $u_{0}^{h}(t)$ is the high-amplitude solution, as shown in Fig. 19(a). On the contrary, if we start from an initial pulse of higher energy, $0.9 u_{0}^{h}(t)$, the CQME evolves to the high-amplitude solution as shown in Fig. 19(b).

The high-amplitude solution remains stable for a very large range of both $\sigma$ and $\delta$. The lower bound of the stability region is shown by curve $C_{2}$ as in Fig. 16, and is bounded on the left by the $\delta$ axis. As we will discuss in detail elsewhere, a selfsimilar solution of the CQME always exists when $\sigma>0$ until $\delta \approx 9.51$, which is larger than the boundary for the lowamplitude solution and, hence, for the HME to become unstable by almost a factor of 280 . The upper bound of the stability region of the high-amplitude solution is the onset of edge bifurcation, followed shortly thereafter as $\delta$ increases by a Hopf bifurcation of the new discrete modes.

We show this stability boundary in Fig. 20, and we see that the boundary for $\delta$ increases slightly as $\sigma$ increases. When this
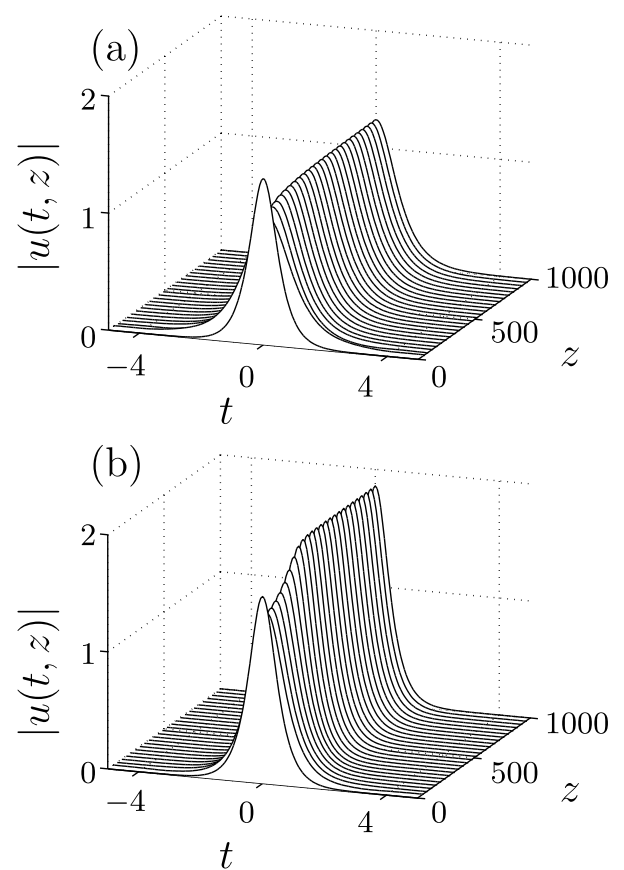

Fig. 19. Evolution of CQME for the case $(\sigma, \delta)=(0.006,0.0413)$. We start from different initial conditions: (a) $0.8 u_{0}^{h}(t)$ and (b) $0.9 u_{0}^{h}(t)$, where $u_{0}^{h}(t)$ is the high-amplitude solution. In the first case, the low-amplitude solution emerges. In the second case, the highamplitude solution emerges.

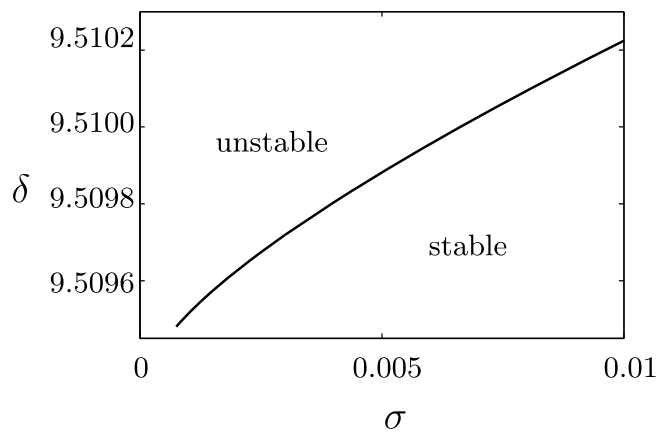

Fig. 20. Stability boundary of the high-amplitude solution due to the edge bifurcation as illustrated in Fig. 12.

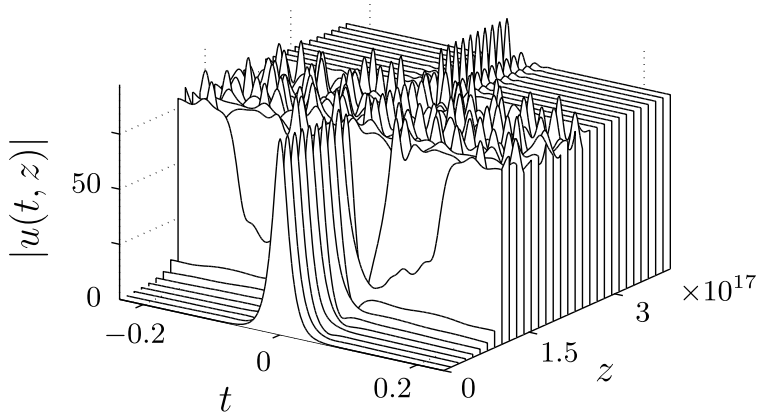

Fig. 21. The pulse evolves to a shelf-like envelope when the eigenmode corresponding to $\lambda_{e}$ becomes unstable.

system becomes unstable, the solution develops a shelf-like envelope, as shown in Fig. 21 for the parameter set $(\sigma=0.003, \delta=13)$. We do not show the stability boundary for $\sigma<7 \times 10^{-3}$ because the equilibrium pulse shape changes rapidly as $\delta$ and $\sigma$ vary, and tracking the boundary becomes computationally time consuming. Indeed, the parameter set at this relatively large value of $\delta$ is sufficiently extreme that it seems unlikely that they correspond to any physical laser system. We present these results here because they illustrate the power of the algorithms that we have developed. The scenario that we have described here in which new discrete modes appear via an edge bifurcation and then become unstable via a Hopf bifurcation appear in practice, for example, when relaxation oscillations appear and then become unstable [38].

\section{CONCLUSION}

We have developed boundary-tracking algorithms that allow us to rapidly and accurately find the stability boundaries in a passively mode-locked laser system as the system parameters vary. We have applied this approach to determining the stability boundaries for the cubic-quintic mode-locking equations as the parameters that govern the staturable absorption, $\sigma$ and $\delta$, are allowed to vary. This model is one of the most commonly used models for passively mode-locked lasers and includes the even more commonly used Haus mode-locking equation as a special limit corresponding to $\sigma=0$.

We have found a rich dynamical structure in which, depending on the parameter, no stable solutions exist, one stable solution exists, or two stable solutions exist. The spectrum of the mode-locked or equilibrium solutions includes both continuous and discrete components. The continuous component 
can become unstable via a Hopf bifurcation and the discrete mode that corresponds to an amplitude change can become unstable via a saddle-node bifurcation. Additionally, we have found that in some extreme parameter ranges, new discrete modes appear in the spectrum, which then become unstable via a Hopf bifurcation.

In future work, we intend to apply these algorithms to increasingly realistic and specialized models of passively modelocked laser systems. So, we will close with a brief discussion of how the algorithms that we have presented here must be modified to be applied correctly to realistic systems. Firstand simplest-are the laser systems in which the CQME is a useful model, but that operate with net zero or normal dispersion. We expect that the methods described in this paper will work equally well in this case. Preliminary work suggests that as we cross from the anomalous to the normal dispersion regime, new discrete modes bifurcate out of the continuous spectrum.

In some solid-state lasers, the gain recovery time is short enough to lead to relaxation oscillations. In this case, the partial differential equations that describe the evolution of the light envelope in the cavity must be supplemented by ordinary differential equations that describe the evolution of the gain [38]. Similarly, modern-day comb lasers include electronic feedback systems that once again lead to equations in which ordinary differential equations are coupled to the partial differential equations that describe the evolution of the light envelope [39]. In both cases, new degrees of freedom are introduced that lead to new discrete modes, but the basic algorithms do not have to be changed.

Somewhat more difficult is dealing with large pulse variations in one round trip in the laser cavity. In this case, the equilibrium is only periodically stationary rather than stationary. Models for analyzing the stability of periodically stationary systems have been developed since the 19th century [7]. In this case, we must calculate the transfer matrix that describe the signals evolution in one round trip. Its equilibrium solutions correspond to the mode-locked pulses, andafter linearization about a solution-its eigenvalues determine the mode-locked pulse's stability. Computational approaches for generating this transfer matrix have been discussed by Holzlöhner et al. [40], as well as Deconinck and Kutz [9].

As a final issue, we may discuss extending this approach to three or more parameters. In a three-parameter space, the stability boundary will become a two-dimensional surface, and tracing the boundary would require a two-dimensional search. On a desktop computer, tracing the stability boundary for the amplitude eigenmode requires a few hours (about four hours in our case) in two parameter dimensions due to the rapid changes of the equilibrium solution at the boundary. By contrast, tracing the other the stability boundaries typically requires less than an hour on a desktop computer, and we have therefore paid little attention to computational optimization. We use standard MATLAB routines and, when finding the discrete eigenmodes, we use MATLAB's eig routine, which generates all the matrix eigenvalues, although we typically need only the amplitude eigenvalue and eigenmode. Clearly, much can be done in the future to speed up the computations, and the speedup will be needed for higher dimensional parameter studies.

\section{APPENDIX A: ON ANALYTICAL SOLUTIONS OF THE CQME}

In this section, we will discuss analytical solutions of the CQME and their relationship to the solutions that we have found computationally in the main text. We will find that the analytical solutions have a special and complicated relation among the parameters, so that a global search for the stability boundaries in which two or more parameters are allowed to vary while all the others are held constant is not possible. We will also find that the chirp parameter $\beta$ must be positive in order for the solution to be stable, which is not the case for the computational solutions that we found.

The CQME is also referred as the complex Ginzburg-Landau equation (CGLE) when the saturated gain in Eq. (2) is replaced by a constant gain $g_{c}$ :

$$
\frac{\partial u}{\partial z}=g_{c} u+\left(\frac{1}{\omega}-\frac{i \beta^{\prime \prime}}{2}\right) \partial_{t}^{2} u+(\delta+i \gamma)|u|^{2} u-\sigma|u|^{4} u,
$$

where $g_{c}$ is a constant gain, $\delta, \gamma$ and $\delta>0$, and $\beta^{\prime \prime}<0$. Comparing to Eq. (1), we make the following correspondence:

$$
g_{c} \leftrightarrow \frac{1}{2}[g(|u|)-l], \frac{1}{\omega} \leftrightarrow \frac{g(|u|)}{2 \omega_{g}^{2}} .
$$

An analytical equilibrium pulse solution of this equation has been be found to exist in certain parameter regimes $[\underline{5}, \underline{12}, \underline{14}, \underline{28}]$ that may be written

$$
\begin{aligned}
u_{0}(t, z)= & \sqrt{\frac{A}{B+\cosh (t / \tau)}} \\
& \times \exp \left\{-\frac{i \beta}{2} \ln \left[B+\cosh \frac{t}{\tau}\right]+i \phi_{0} z\right\},
\end{aligned}
$$

where $A, B, \beta$, and $\tau$ can be written in terms of the coefficients in Eq. (A1). We may choose $A>0$ to avoid the ambiguity of the sign of the square root. With this choice, we find $B+\cosh (t / \tau)>0$, which implies $B>-1$.

We define

$$
a(t)=[B+\cosh (t / \tau)]^{-1 / 2},
$$

and substitute $a(t)$ into Eq. (A1). Equating the coefficients of $a$ of the same power, we obtain the following three equations:

$$
\begin{gathered}
g_{c}-i \phi+\frac{1}{4 \tau^{2}}\left(\frac{1}{\omega}-\frac{i \beta^{\prime \prime}}{2}\right)\left(1-\beta^{2}+2 i \beta\right)=0, \\
(\delta+i \gamma) A-\frac{1}{2 \tau^{2}}\left(\frac{1}{\omega}-\frac{i \beta^{\prime \prime}}{2}\right)\left(2-\beta^{2}+3 i \beta\right) B=0, \\
\frac{1}{4 \tau^{2}}\left(\frac{1}{\omega}-\frac{i \beta^{\prime \prime}}{2}\right)\left(3-\beta^{2}+4 i \beta\right)\left(B^{2}-1\right)-\sigma A^{2}=0 .
\end{gathered}
$$

By splitting Eq. (A5) into real and imaginary parts, we obtain

$$
4 g_{c} \omega \tau^{2}+1-\beta^{2}+\beta^{\prime \prime} \omega \beta=0,
$$




$$
\begin{gathered}
8 \phi_{0} \tau^{2} \omega-4 \beta+\beta^{\prime \prime} \omega\left(1-\beta^{2}\right)=0, \\
2\left(\beta^{2}-2\right) B-3 \beta^{\prime \prime} \omega \beta B+4 \delta A \tau^{2} \omega=0, \\
-6 \beta B+\beta^{\prime \prime} \omega B\left(2-\beta^{2}\right)+4 \gamma A \tau^{2} \omega=0, \\
\left(B^{2}-1\right)\left(3-\beta^{2}+2 \beta^{\prime \prime} \beta \omega\right)-4 \sigma A^{2} \tau^{2} \omega=0, \\
\left(B^{2}-1\right)\left[8 \beta+\beta^{\prime \prime} \omega\left(\beta^{2}-3\right)\right]=0 .
\end{gathered}
$$

Equation (A6f) implies that there are two cases: $B^{2} \neq 1$ and $B^{2}=1$. If $B^{2}=1$, then Eq. (A6e) implies that the system has a solution when $\sigma=0$. This solution corresponds to a modelocking equation that only has a cubic nonlinearity [28] and whose known analytical solutions are chirped hyperbolic secant pulses [28]. These solutions exist only when a special relation among the coefficients of Eq. (A1) holds.

We now consider the case $B \neq 1$ in the parameter regime $\beta^{\prime \prime}<0$. We introduce an intermediate variable $\Delta$ as in [12]:

$$
\Delta=\sqrt{3 \beta^{\prime \prime 2} \omega^{2}+16}
$$

Then, using Eq. (A6f) we can write the exponential chirp $\beta$ as

$$
\beta \pm=\frac{4 \pm \Delta}{\beta^{\prime \prime} \omega} .
$$

We next write all the pulse parameters in terms of $\beta$ and the

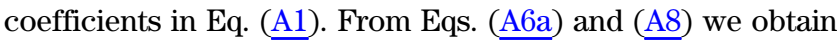

$$
\tau=\sqrt{-\frac{\beta^{\prime \prime}\left(\beta^{2}+1\right)\left(\beta^{2}+3\right)}{32 g_{c} \beta} .}
$$

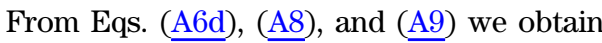

$$
A=-\frac{2 \beta g_{c} B}{\gamma\left(\beta^{2}+3\right)},
$$

and from Eqs. (

$$
B^{2}=\frac{\gamma^{2}\left(\beta^{2}+3\right)\left(\beta^{2}+9\right)}{\gamma^{2}\left(\beta^{2}+3\right)\left(\beta^{2}+9\right)+4 \sigma g_{c} \beta^{2}} .
$$

In theory, we can rewrite $A$ by combining Eqs. (A10) and (A11) directly. If we do not put any limit on the coefficients of Eq. (A1), the sign of $B$ can be either positive or negative, which leads to two different expressions for $A$ and, hence, two branches of solutions [5]. We do not further elaborate them.

We now take a close look at this analytical solution. From Eqs. ( $\underline{\mathrm{A} 6 \mathrm{c})}$ ) and $(\underline{\mathrm{A} 6 \mathrm{~d})}$, we obtain

$$
\frac{2\left(\beta^{2}-2\right)-3 \beta^{\prime \prime} \omega \beta}{\delta}=\frac{\beta^{\prime \prime} \omega\left(2-\beta^{2}\right)-6 \beta}{\gamma} .
$$

Meanwhile, from Eq. (A6f), we obtain

$$
\omega=\frac{8 \beta}{\beta^{\prime \prime}\left(3-\beta^{2}\right)} .
$$

Combining Eq. ( $\underline{\mathrm{A} 12})$ and ( $\underline{\mathrm{A} 13})$, we finally obtain

$$
\frac{\delta}{\gamma}=\frac{\beta^{2}+6}{\beta} .
$$

So, we conclude that for this solution to exist, it is required that $\beta>0$ and

$$
\beta=\frac{4-\Delta}{\beta^{\prime \prime} \omega} .
$$

From Eqs. (14) and (A15), we have

$$
\frac{\delta}{\gamma}=\frac{32+9 \beta^{\prime \prime 2} \omega^{2}-8 \sqrt{16+3 \beta^{\prime \prime 2} \omega^{2}}}{\beta^{\prime \prime 3} \omega^{3}} .
$$

Hence, the coefficients of CGLE must have a special relation for this analytical solution to exist. However, for cases where Eq. (A16) is not satisfied, such as in the CQME, one can still find equilibrium solutions computationally, which implies the existence of more general solutions, for which no analytical solutions are known.

If we require $\beta>0$, we must have $g_{c}>0$ from Eq. (A9) since $\beta^{\prime \prime}<0$, which indicates that this solution is unstable to modes of the continuous spectrum according to the inequalities in Eqs. (24) and (A2). However, we have found that this statement is not true for the numerical solutions that we have found in the text. Therefore, we can conclude that numerical techniques are needed to study the stability of Eq. (1) and more complex models.

\section{APPENDIX B: NUMERICAL EVALUATION OF THE CONTINUOUS EIGENVALUES}

The computational requirement that $T \ll T_{R}$ poses no difficulty for discrete modes except immediately after an edge bifurcation, since these modes rapidly tend to zero away from the mode-locked pulse. However, it does pose a problem for the modes of the continuous spectrum. We dealt with this problem in the main text by using the same infinite-line approximation that is used in analytical studies of the CQME that is described in Section 4.B.1. This approach allows us to determine the stability of the continuous spectrum by studying the dispersion relation away from the mode-locked pulse. We could in principle use an approach analogous to the approach that we used to study edge bifurcations, where we would assume that beyond a limited time window the mode undergoes sinusoidal oscillations that repeat with a period $T_{R}$. However, this approach would be computationally inefficient. It is thus reasonable to ask on one hand how large $T_{R}$ must be for the eigenvalues of the discrete radiation modes that we found in a time window of duration $T$ to converge to the continuous spectrum. They must converge for $T \ll T_{R}$ in order for our approach to be legitimate. On the other hand, it is also reasonable to ask whether it is really necessary to use different algorithms to compute the stability of modes of the continuous spectrum and the discrete modes, or whether it is possible to simply use the spectrum of the radiation modes that are calculated using a limited time window. 


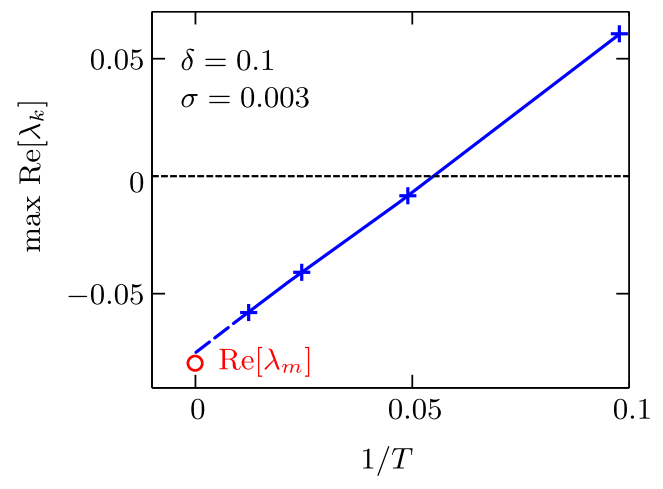

Fig. 22. The maximum of the real part of the spectrum calculated numerically using periodic boundary conditions deviates from that of the spectrum derived on the infinite time axis using Eq. (23). The duration of the equilibrium pulse $\tau \approx 0.204$ is calculated using Eq. (12), and $\lambda_{m}=(g-l+i \phi) / 2$ is the maximum of the continuous spectrum according to Eq. (23) when $\omega=0$.

In Fig. 22, we show how the maximum of the real part of the entire continuous spectrum, which is obtained by solving the eigenvalue problem that is described in Section 4.B.2, changes as the duration of the time window $T$ varies. We have set $\delta=$ 0.1 and $\sigma=0.003$ in this plot, but we have verified that the results are typical in the range shown in Fig. 16. The discrete radiation spectrum has two branches, which has been previously observed in computational solutions of the HME [9]. We see that $\max [\operatorname{Re}(\lambda)]$ only converges slowly to the continuum value, and is positive until $T>90 \tau$, even though the true value is negative. We note that, if one simply solved the evolution equations, starting from computational noise with a window $\sim 10 \tau$, as is often done in practice, one would observe unstable behavior and incorrectly predict that the laser system is unstable.

Convergence to the maximum of the real part of the infiniteline continuous spectrum is slow as $\tau \rightarrow 0$ and appears to be nonanalytic in $1 / T$. The reason is that the most unstable (or least stable) mode keeps changing as $T$ increases. We find that, with a time window that is 2000 times the pulse duration, the least stable eigenvalue differs from the infinite-line value by about $10 \%$. This result has important consequences for modeling high-repetition-rate lasers $(\sim 10 \mathrm{GHz})$ or microresonators, where an infinite line model is often used in analytical studies, but is almost certainly invalid when making predictions of the systems' stability.

\section{ACKNOWLEDGMENTS}

This research was supported in its final stage by the DARPA PULSE program via AMRDEC.

\section{REFERENCES}

1. H. A. Haus, "Mode-locking of lasers," IEEE J. Sel. Top. Quantum Electron. 6, 1173-1185 (2000).

2. M. E. Fermann, "Ultrafast fiber oscillators," in Ultrafast Lasers: Technology and Applications, M. E. Fermann, A. Galvanauskas, and G. Sucha, eds. (Marcel Dekker, 2002), Chap. 3.

3. F. Kärtner, U. Morgner, T. Schibli, R. Ell, H. A. Haus, J. Fujimoto, and E. Ippen, "Few-cycle pulses directly from a laser," in FewCycle Laser Pulse Generation and Its Applications, F. Kärtner, ed., Topics in Applied Physics (Springer, 2004), pp. 73-136.

4. J. Soto-Crespo, N. N. Akhmediev, and K. S. Chiang, "Simultaneous existence of a multiplicity of stable and unstable solitons in dissipative systems," Phys. Lett. A 291, 115-123 (2001).
5. J. M. Soto-Crespo, N. N. Akhmediev, and V. V. Afanasjev, "Stability of the pulselike solutions of the quintic complex GinzburgLandau equation," J. Opt. Soc. Am. B 13, 1439-1449 (1996).

6. N. N. Akhmediev, J. M. Soto-Crespo, and P. Grelu, "Roadmap to ultra-short record high-energy pulses out of laser oscillators," Phys. Lett. A 372, 3124-3128 (2008).

7. S. Strogatz, Nonlinear Dynamics and Chaos: With Applications to Physics, Biology, Chemistry and Engineering, Studies in Nonlinearity Series (Perseus, 1994).

8. M. Hirsch, S. Smale, and R. Devaney, Differential Equations, Dynamical Systems, and an Introduction to Chaos (Academic, 2004).

9. B. Deconinck and J. N. Kutz, "Computing spectra of linear operators using the Floquet-Fourier-Hill method," J. Comput. Phys. 219, 296-321 (2006).

10. C.-J. Chen, P. K. A. Wai, and C. R. Menyuk, "Stability of passively mode-locked fiber lasers with fast saturable absorption," Opt. Lett. 19, 198-200 (1994).

11. H. A. Haus, "Theory of mode locking with a fast saturable absorber,” J. Appl. Phys. 46, 3049-3058 (1975).

12. W. H. Renninger, A. Chong, and F. Wise, "Dissipative solitons in normal-dispersion fiber lasers," Phys. Rev. A 77, 023814 (2008).

13. J. N. Kutz, "Mode-locked soliton lasers," SIAM Rev. 48, 629-678 (2006).

14. M. Romagnoli, S. Wabnitz, P. Franco, M. Midrio, L. Bossalini, and F. Fontana, "Role of dispersion in pulse emission from a slidingfrequency fiber laser," J. Opt. Soc. Am. B 12, 938-944 (1995).

15. T. Kapitula, J. N. Kutz, and B. Sandstede, "Stability of pulses in the master mode-locking equation," J. Opt. Soc. Am. B 19, $740-746$ (2002)

16. T. Kapitula, J. N. Kutz, and B. Sandstede, "The Evans function for nonlocal equations," Indiana Univ. Math. J. 53, 1095-1126 (2004).

17. H. Poincaré, Les méthodes nouvelles de la mécanique céleste (Gauthier-Villars et fils, 1899).

18. L. D. Landau and E. M. Lifshitz, The Classical Theory of Fields, 2nd ed., Course of Theoretical Physics (Pergamon, 1962) [Translated from Russian].

19. L. D. Landau and E. M. Lifshitz, Fluid Mechanics, 2nd ed., Course of Theoretical Physics (Butterworth-Heinemann, 1987), Chap. 3 [translated from Russian].

20. F. X. Kärtner, I. Jung, and U. Keller, "Soliton mode-locking with saturable absorbers," IEEE J. Sel. Top. Quantum Electron. 2 540-556 (1996).

21. T. Kapitula and B. Sandstede, "Edge bifurcations for near integrable systems via Evans function techniques," SIAM J. Math. Anal. 33, 1117-1143 (2002).

22. J. Hu and C. R. Menyuk, "Understanding leaky modes: slab waveguide revisited," Adv. Opt. Photon. 1, 58-106 (2009).

23. E. Podivilov and V. L. Kalashnikov, "Heavily-chirped solitary pulses in the normal dispersion region: new solutions of the cubic-quintic complex Ginzburg-Landau equation," J. Exp. Theor. Phys. 82, 467-471 (2005).

24. V. L. Kalashnikov, E. Podivilov, A. Chernykh, and A. Apolonski, "Chirped-pulse oscillators: theory and experiment," Appl. Phys. B 83, 503-510 (2006).

25. V. L. Kalashnikov and A. Apolonski, "Chirped-pulse oscillators: a unified standpoint," Phys. Rev. A 79, 043829 (2009).

26. V. L. Kalashnikov, "Chirped dissipative solitons of the complex cubic-quintic nonlinear Ginzburg-Landau equation," Phys. Rev. E 80, 046606 (2009).

27. D. S. Kharenko, O. V. Shtyrina, I. A. Yarutkina, E. V. Podivilov, M. P. Fedoruk, and S. A. Babin, "Highly chirped dissipative solitons as a one-parameter family of stable solutions of the cubic-quintic Ginzburg-Landau equation,” J. Opt. Soc. Am. B 28, 2314-2319 (2011).

28. H. A. Haus and A. Mecozzi, "Noise of mode-locked lasers," IEEE J. Quantum Electron. 29, 983-996 (1993).

29. D. J. Kaup, "Perturbation theory for solitons in optical fibers," Phys. Rev. A 42, 5689-5694 (1990).

30. S. A. Diddams, "The evolving optical frequency comb [Invited]," J. Opt. Soc. Am. B 27, B51-B62 (2010). 
31. J. K. Wahlstrand, J. T. Willits, C. R. Menyuk, and S. T. Cundiff, "The quantum-limited comb lineshape of a mode-locked laser: fundamental limits on frequency uncertainty," Opt. Express 16, 18624-18630 (2008).

32. S. Wang, A. Docherty, B. S. Marks, and C. R. Menyuk, "Comparison of numerical methods for modeling laser mode locking with saturable gain," J. Opt. Soc. Am. B 30, 3064-3074 (2013).

33. R. Paschotta, "Noise of mode-locked lasers (part I): numerical model," Appl. Phys. B 79, 153-162 (2004).

34. J. Nocedal and S. Wright, Numerical Optimization, Springer Series in Operations Research and Financial Engineering (Springer, 2006).

35. E. Ding and J. N. Kutz, "Operating regimes, split-step modeling, and the Haus master mode-locking model," J. Opt. Soc. Am. B 26, 2290-2300 (2009).
36. B. Sandstede and A. Scheel, "Essential instability of pulses and bifurcations to modulated travelling waves," Proc. Roy. Soc. Edinburgh Sect. A 129, 1263-1290 (1999).

37. J. A. C. Weideman and S. C. Reddy, "A MATLAB differentiation matrix suite," ACM Trans. Math. Softw. 26, 465-519 (2000).

38. C. R. Menyuk, J. K. Wahlstrand, J. Willits, R. P. Smith, T. R. Schibli, and S. T. Cundiff, "Pulse dynamics in mode-locked lasers: relaxation oscillations and frequency pulling," Opt. Express 15, 6677-6689 (2007).

39. M. Shtaif, C. R. Menyuk, M. L. Dennis, and M. C. Gross, "Carrierenvelope phase locking of multi-pulse lasers with an intra-cavity Mach-Zehnder interferometer," Opt. Express 19, 23202-23214 (2011).

40. R. Holzlöhner, C. R. Menyuk, W. L. Kath, and V. S. Grigoryan, "A covariance matrix method to compute bit error rates in a highly nonlinear dispersion-managed soliton system," IEEE Photon Technol. Lett. 15, 688-690 (2003). 